\title{
Stability of discrete-time HIV dynamics models with long- lived chronically infected cells
}

\author{
A. M. Elaiw ${ }^{a, *}$, M. A. Alshaikh ${ }^{a, b}$ \\ ${ }^{a}$ Department of Mathematics, Faculty of Science, King Abdulaziz University, P. O. Box 80203, Jeddah 21589, Saudi Arabia. \\ ${ }^{b}$ Department of Mathematics, Faculty of Science, Taif University, Saudi Arabia.
}

\begin{abstract}
This paper studies the global dynamics for discrete-time HIV infection models. The models integrate both long-lived chronically infected and short-lived infected cells. The HIV-susceptible incidence rate is taken as bilinear, saturation and general function. We discretize the continuous-time models by using nonstandard finite difference scheme. The positivity and boundedness of solutions are established. The basic reproduction number is derived. By using Lyapunov method, we prove the global stability of the models. Numerical simulations are presented to illustrate our theoretical results.
\end{abstract}

Keywords: HIV infection, short-lived infected cells, long-lived infected cells, global stability, Lyapunov function.

2010 MSC: 34D20, 34D23, 37N25, 92B05.

(C)2019 All rights reserved.

\section{Introduction}

Human immunodeficiency virus (HIV) destroys the human immune system by attacking the CD4 ${ }^{+} \mathrm{T}$ cells. Modeling within host dynamics of HIV has attracted the interest of several researchers (see, e.g., [1-3, 6-14, 19, 23, 26, 27, 32-34, 40, 41, 47]). The basic HIV dynamics model is given by [33]:

$$
\dot{\mathrm{s}}=\beta-\delta \mathrm{s}-\overline{\mathrm{ksp}}, \quad \dot{\mathrm{z}}=\overline{\mathrm{k}} \mathrm{sp}-\mathrm{d} z, \quad \dot{\mathrm{p}}=\mathrm{N}_{z} \mathrm{~d} z-\mathrm{cp},
$$

where $s, z$ and $p$ are the concentrations of susceptible (uninfected) CD4 ${ }^{+} \mathrm{T}$ cells, infected cells, and free HIV particles, respectively. Parameters $\beta, \delta$, and $\bar{k}$ represent the rate of birth, death, and infection of the susceptible cells, respectively. The parameters $d$ and $c$ are the death rate constants of the infected cells and free HIV particles, respectively. $\mathrm{N}_{z}$ is the average number of HIV particles produced in the lifetime of the infected cell.

Antiretroviral drug therapies such as reverse transcriptase inhibitors (RTI) and protease inhibitors (PI) can significantly reduce the level of HIV in the blood. However, there still is a low viral load due to the

\footnotetext{
${ }^{*}$ Corresponding author

Email addresses: a_m_elaiw@yahoo.com (A. M. Elaiw), matukaalshaikh@gmail.com (M. A. Alshaikh)

doi: $10.22436 /$ jnsa.012.07.02
}

Received: 2018-11-16 Revised: 2018-12-19 Accepted: 2019-01-14 
presence of long-lived chronically infected cells. Callaway and Perelson [1] have proposed the following HIV dynamics model with both short-lived infected and long-lived chronically infected cells:

$$
\begin{aligned}
& \dot{\mathrm{s}}=\beta-\delta s-(1-\epsilon) \bar{k} s p \\
& \dot{z}=(1-\alpha)(1-\epsilon) \bar{k} s p-d z \\
& \dot{u}=\alpha(1-\epsilon) \bar{k} s p-a u, \\
& \dot{p}=N_{z} d z+N_{u} a u-c p
\end{aligned}
$$

where $z$ and $u$ are the concentrations of short-lived infected cells and long-lived chronically infected cells. A fraction $(1-\alpha)$ and $\alpha$ with $0<\alpha<1$ are the probabilities that, upon infection, susceptible cell will become either short-lived infected or chronically infected. The parameter $a$ is the death rate constant of the chronically infected cells. $N_{\mathfrak{u}}$ is the average number of HIV particles produced in the lifetime of the chronically infected cells. The chronically infected cells produce much smaller amounts of HIV than the short-lived infected cells and die at a much slower rate [1]. The RTI drug efficacy is denoted by $\epsilon$ and $0 \leqslant \epsilon \leqslant 1$.

Model (1.1)-(1.4) has been described by system of nonlinear ODEs, but the exact analytical solution of the model is unknown. Therefore, a discretization can be used to obtain discrete-time model which is an approximation of the exact one. Further, the use of digital computers in performing simulations necessitated the investigation of discrete-time systems. Furthermore, it is important to note that scientists often collect the data and analyze the results at discrete times. One of the very important task is to choose a discretization scheme which preserves the properties of the corresponding continuous time model. In 1994 Mickens [30] has introduced nonstandard finite difference (NSFD) scheme for solving differential equations. It has been proven that NSFD can preserve the main properties of several types of continuous time models. NSFD has been used to investigate the global stability of equilibria of the corresponding continuous time models in epidemiology $[4,5,16,18,28,39]$ and virology $[17,20-22,24,29,35,36,41-$ $46,48]$.

In this paper, our target is to study a class of discrete time HIV infection models with both short-lived infected and long-lived chronically infected cells. We study the qualitative behavior of the models with different forms of the infection rate. We investigate global stability of the equilibria of the models using Lyapunov method.

\section{Discrete-time model}

Discretizing system (1.1)-(1.4) using NSFD method as in [30] and [31] we obtain

$$
\begin{aligned}
s_{n+1}-s_{n} & =\beta-\delta s_{n+1}-k s_{n+1} p_{n} \\
z_{n+1}-z_{n} & =(1-\alpha) k s_{n+1} p_{n}-d z_{n+1} \\
u_{n+1}-u_{n} & =\alpha k s_{n+1} p_{n}-a u_{n+1} \\
p_{n+1}-p_{n} & =N_{z} d z_{n+1}+N_{u} a u_{n+1}-c p_{n+1}
\end{aligned}
$$

where, $k=(1-\epsilon) \bar{k}$ and $n \in \mathbb{N}=\{0,1,2, \ldots\}$. We consider the initial conditions:

$$
\left(s_{0}, z_{0}, u_{0}, p_{0}\right) \in \mathbb{R}_{+}^{4}=\{(s, z, u, p) \mid s>0, z>0, u>0, p>0\} .
$$

\subsection{Preliminaries}

Let us consider the region

$$
\Gamma_{1}=\left\{(s, z, u, p): 0<s, z, u<N_{1}, 0<p<N_{2}\right\},
$$

where $N_{1}=\frac{\beta}{\xi}, N_{2}=\frac{\left(N_{z} \lambda+N_{u} a\right)}{c} N_{1}$ and $\xi=\min \{\delta, d, a\}$. 
Lemma 2.1. Any solution $\left(s_{n}, z_{n}, u_{n}, p_{n}\right)$ of model (2.1)-(2.4) with initial condition (2.5) is positive and ultimately bounded.

Proof. From Eqs. (2.1)-(2.4) we obtain

$$
\begin{aligned}
s_{n+1} & =\frac{\beta+s_{n}}{1+\delta+k p_{n}}, \\
z_{n+1} & =\frac{z_{n}}{1+d}+\frac{(1-\alpha) k\left(\beta+s_{n}\right) p_{n}}{(1+d)\left(1+\delta+k p_{n}\right)}, \\
u_{n+1} & =\frac{u_{n}}{1+a}+\frac{\alpha k\left(\beta+s_{n}\right) p_{n}}{(1+a)\left(1+\delta+k p_{n}\right)}, \\
p_{n+1} & =\frac{p_{n}}{1+c}+\frac{N_{z} d}{1+c}\left(\frac{z_{n}}{1+d}+\frac{(1-\alpha) k\left(\beta+s_{n}\right) p_{n}}{(1+d)\left(1+\delta+k p_{n}\right)}\right)+\frac{N_{u} a}{1+c}\left(\frac{u_{n}}{1+a}+\frac{\alpha k\left(\beta+s_{n}\right) p_{n}}{(1+a)\left(1+\delta+k p_{n}\right)}\right) .
\end{aligned}
$$

Since all parameters in (2.1)-(2.4) and the initial condition (2.5) are positive, then by induction we get $s_{n}>0, z_{n}>0, u_{n}>0$ and $p_{n}>0$ for all $n \in \mathbb{N}$.

Define a sequence $M_{n}$ as:

$$
M_{n}=s_{n}+z_{n}+u_{n}
$$

Then

$$
M_{n+1}=M_{n}+\beta-\delta s_{n+1}-d z_{n+1}-a u_{n+1} \leqslant M_{n}+\beta-\xi M_{n+1},
$$

Hence

$$
M_{n+1} \leqslant \frac{M_{n}}{1+\xi}+\frac{\beta}{1+\xi}
$$

According Lemma 2.2 in [36] we obtain

$$
M_{n} \leqslant\left(\frac{1}{1+\xi}\right)^{n} M_{0}+\frac{\beta}{\xi}\left[1-\left(\frac{1}{1+\xi}\right)^{n}\right] .
$$

Consequently, $\lim _{n \rightarrow \infty} \sup M_{n} \leqslant N_{1}, \lim _{n \rightarrow \infty} \sup s_{n} \leqslant N_{1}, \lim _{n \rightarrow \infty} \sup z_{n} \leqslant N_{1}, \lim _{n \rightarrow \infty} \sup u_{n} \leqslant N_{1}$. We have

$$
p_{n+1}-p_{n}=N_{z} d z_{n+1}+N_{u} a u_{n+1}-c p_{n+1} \leqslant\left(N_{z} d+N_{u} a\right) \frac{\beta}{\xi}-c p_{n+1}
$$

Hence

$$
p_{n+1} \leqslant \frac{p_{n}}{1+c}+\frac{\left(N_{z} d+N_{u} a\right)}{(1+c)} \frac{\beta}{\xi}=\frac{p_{n}}{1+c}+\frac{\left(N_{z} d+N_{u} a\right)}{(1+c)} N_{1}
$$

By induction

$$
p_{n} \leqslant\left(\frac{1}{1+c}\right)^{n} p_{0}+\frac{\left(N_{z} \lambda+N_{u} a\right) \beta}{c \xi}\left[1-\left(\frac{1}{1+c}\right)^{n}\right]
$$

Consequently, $\lim _{n \rightarrow \infty} \sup p_{n} \leqslant N_{2}$. Therefore, the solution $\left(s_{n}, z_{n}, u_{n}, p_{n}\right)$ converges to $\Gamma_{1}$ as $n \rightarrow \infty$.

System (2.1)-(2.4) has two equilibria,

(i) HIV-free equilibrium $\mathrm{Q}^{0}\left(s^{0}, 0,0,0\right)$, where $s^{0}=\beta / \delta$;

(ii) persistent HIV equilibrium $\mathrm{Q}^{*}\left(\mathrm{~s}^{*}, z^{*}, \mathrm{u}^{*}, \mathrm{p}^{*}\right)$, where

$$
s^{*}=\frac{s^{0}}{\mathcal{R}_{0}}, \quad z^{*}=\frac{\beta(1-\alpha)}{\mathrm{d} \mathcal{R}_{0}}\left(\mathcal{R}_{0}-1\right), \quad u^{*}=\frac{\alpha \beta}{\mathrm{a} \mathcal{R}_{0}}\left(\mathcal{R}_{0}-1\right), \quad p^{*}=\frac{\delta}{\mathrm{k}}\left(\mathcal{R}_{0}-1\right) .
$$

Clearly, $\mathrm{Q}^{*}$ exists only when $\mathcal{R}_{0}>1$, where $\mathcal{R}_{0}$ is basic reproduction number and is given by:

$$
\mathcal{R}_{0}=\frac{k \beta}{\delta \mathrm{c}}\left(\mathrm{N}_{z}(1-\alpha)+\alpha \mathrm{N}_{\mathrm{u}}\right) .
$$




\subsection{Global Stability}

We define the function $G(x) \geqslant 0$ as $G(x)=x-\ln x-1$. Hence,

$$
\ln x \leqslant x-1
$$

Theorem 2.2. If $\mathcal{R}_{0} \leqslant 1$, then $\mathrm{Q}^{0}$ is globally asymptotically stable.

Proof. Construct a discrete Lyapunov function $\mathrm{L}_{n}\left(\mathrm{~s}_{n}, z_{n}, \mathrm{u}_{n}, \mathrm{p}_{n}\right)$ as:

$$
L_{n}=s^{0} G\left(\frac{s_{n}}{s^{0}}\right)+\eta_{1} z_{n}+\eta_{2} u_{n}+\eta_{3}(1+c) p_{n},
$$

where $\eta_{i}, i=1,2,3$ are positive constants to be determined below. Hence, $L_{n}>0$ for all $s_{n}>0, z_{n}>0$, $u_{n}>0$ and $p_{n}>0$. In addition, $L_{n}=0$ if and only if $s_{n}=s^{0}, z_{n}=0, u_{n}=0$ and $p_{n}=0$. Computing the difference $\Delta \mathrm{L}_{\mathrm{n}}=\mathrm{L}_{\mathrm{n}+1}-\mathrm{L}_{\mathrm{n}}$, as:

$$
\begin{aligned}
\Delta L_{n} & =s^{0} G\left(\frac{s_{n+1}}{s^{0}}\right)+\eta_{1} z_{n+1}+\eta_{2} u_{n+1}+\eta_{3}(1+c) p_{n+1}-\left[s^{0} G\left(\frac{s_{n}}{s^{0}}\right)+\eta_{1} z_{n}+\eta_{2} u_{n}+\eta_{3}(1+c) p_{n}\right] \\
& =s^{0}\left(\frac{s_{n+1}}{s^{0}}-\frac{s_{n}}{s^{0}}+\ln \frac{s_{n}}{s_{n+1}}\right)+\eta_{1}\left(z_{n+1}-z_{n}\right)+\eta_{2}\left(u_{n+1}-u_{n}\right)+\eta_{3}(1+c)\left(p_{n+1}-p_{n}\right) .
\end{aligned}
$$

Using inequality (2.7), we have

$$
\begin{aligned}
\Delta L_{n} & \leqslant s_{n+1}-s_{n}+s^{0}\left(\frac{s_{n}}{s_{n+1}}-1\right)+\eta_{1}\left(z_{n+1}-z_{n}\right)+\eta_{2}\left(u_{n+1}-u_{n}\right)+\eta_{3}(1+c)\left(p_{n+1}-p_{n}\right) \\
& =\left(1-\frac{s^{0}}{s_{n+1}}\right)\left(s_{n+1}-s_{n}\right)+\eta_{1}\left(z_{n+1}-z_{n}\right)+\eta_{2}\left(u_{n+1}-u_{n}\right)+\eta_{3}(1+c)\left(p_{n+1}-p_{n}\right) .
\end{aligned}
$$

From Eqs. (2.1)-(2.4), we have

$$
\begin{aligned}
\Delta \mathrm{L}_{n} \leqslant & \left(1-\frac{s^{0}}{s_{n+1}}\right)\left(\beta-\delta s_{n+1}-k s_{n+1} p_{n}\right)+\eta_{1}\left((1-\alpha) k s_{n+1} p_{n}-d z_{n+1}\right) \\
& +\eta_{2}\left(\alpha k s_{n+1} p_{n}-a u_{n+1}\right)+\eta_{3}\left(N_{z} d z_{n+1}+N_{u} a u_{n+1}-c p_{n+1}\right)+\eta_{3} c\left(p_{n+1}-p_{n}\right) .
\end{aligned}
$$

Let $\eta_{i}, i=1,2,3$, be chosen such as

$$
(1-\alpha) \eta_{1}+\alpha \eta_{2}=1, \quad \mathrm{~N}_{z} \eta_{3}-\eta_{1}=0, \quad \mathrm{~N}_{\mathfrak{u}} \eta_{3}-\eta_{2}=0 .
$$

The solution of (2.8) is given by

$$
\eta_{1}=\frac{N_{z}}{\alpha N_{u}+(1-\alpha) N_{z}}, \quad \eta_{2}=\frac{N_{u}}{\alpha N_{u}+(1-\alpha) N_{z}}, \quad \eta_{3}=\frac{1}{\alpha N_{\mathfrak{u}}+(1-\alpha) N_{z}} .
$$

The values of $\eta_{i}, i=1,2,3$, given by (2.9) will be used thought the paper. Then

$$
\begin{aligned}
\Delta \mathrm{L}_{n} & \leqslant\left(1-\frac{s^{0}}{s_{n+1}}\right)\left(\beta-\delta s_{n+1}\right)+k s^{0} p_{n}-\eta_{3} c p_{n} \\
& =\frac{-\delta}{s_{n+1}}\left(s_{n+1}-s^{0}\right)^{2}+\left(k s^{0}-\eta_{3} c\right) p_{n} \\
& =\frac{-\delta}{s_{n+1}}\left(s_{n+1}-s^{0}\right)^{2}+\eta_{3} c\left(\frac{k s^{0}\left(\alpha N_{u}+(1-\alpha) N_{z}\right)}{c}-1\right) p_{n} \\
& =\frac{-\delta}{s_{n+1}}\left(s_{n+1}-s^{0}\right)^{2}+\eta_{3} c\left(\mathcal{R}_{0}-1\right) p_{n} .
\end{aligned}
$$


Hence, for $\mathcal{R}_{0} \leqslant 1$, we have $\Delta \mathrm{L}_{n} \leqslant 0$ for all $\mathrm{n} \geqslant 0$, hence $\mathrm{L}_{\mathrm{n}}$ is a monotone decreasing sequence. we have $\mathrm{L}_{n} \geqslant 0$, then there is a limit $\lim _{n \rightarrow \infty} \mathrm{L}_{n} \geqslant 0$. Therefore, $\lim _{n \rightarrow \infty} \Delta \mathrm{L}_{n}=0$, which implies that $\lim _{n \rightarrow \infty} s_{n+1}=s^{0}$ and $\lim _{n \rightarrow \infty}\left(\mathcal{R}_{0}-1\right) p_{n}=0$. For the case $\mathcal{R}_{0}<1$, we have $\lim _{n \rightarrow \infty} s_{n+1}=s^{0}$ and $\lim _{n \rightarrow \infty} p_{n}=0$. From Eqs. (2.2)-(2.4), we obtain $\lim _{n \rightarrow \infty} z_{n}=0$ and $\lim _{n \rightarrow \infty} u_{n}=0$. For the case $\mathcal{R}_{0}=1$, we have $\lim _{n \rightarrow \infty} s_{n+1}=s^{0}$. From Eqs. (2.2)-(2.4), we obtain $\lim _{n \rightarrow \infty} p_{n}=0, \lim _{n \rightarrow \infty} u_{n}=0$ and $\lim _{n \rightarrow \infty} z_{n}=0$. Hence, in the case $\mathcal{R}_{0} \leqslant 1$, the HIV-free equilibrium $\mathrm{Q}^{0}$ is globally asymptotically stable.

Theorem 2.3. If $\mathcal{R}_{0}>1$, then $\mathrm{Q}^{*}$ is globally asymptotically stable.

Proof. Define

$$
\mathrm{U}_{n}\left(s_{n}, z_{n}, u_{n}, p_{n}\right)=s^{*} G\left(\frac{s_{n}}{s^{*}}\right)+\eta_{1} z^{*} G\left(\frac{z_{n}}{z^{*}}\right)+\eta_{2} u^{*} G\left(\frac{u_{n}}{u^{*}}\right)+(1+c) \eta_{3} p^{*} G\left(\frac{p_{n}}{p^{*}}\right),
$$

where $\eta_{i}, i=1,2,3$ are given by Eq. (2.9). Clearly, $u_{n}\left(s_{n}, z_{n}, u_{n}, p_{n}\right)>0$ for all $s_{n}, z_{n}, u_{n}, p_{n}>0$ and $\mathrm{U}_{\mathrm{n}}\left(\mathrm{s}^{*}, \mathrm{z}^{*}, \mathrm{u}^{*}, \mathrm{p}^{*}\right)=0$. Computing $\Delta \mathrm{U}_{\mathrm{n}}=\mathrm{U}_{\mathrm{n}+1}-\mathrm{U}_{\mathrm{n}}$ as:

$$
\begin{aligned}
\Delta u_{n}= & s^{*} G\left(\frac{s_{n+1}}{s^{*}}\right)+\eta_{1} z^{*} G\left(\frac{z_{n+1}}{z^{*}}\right)+\eta_{2} u^{*} G\left(\frac{u_{n+1}}{u^{*}}\right)+(1+c) \eta_{3} p^{*} G\left(\frac{p_{n+1}}{p^{*}}\right) \\
& -\left[s^{*} G\left(\frac{s_{n}}{s^{*}}\right)+\eta_{1} z^{*} G\left(\frac{z_{n}}{z^{*}}\right)+\eta_{2} u^{*} G\left(\frac{u_{n}}{u^{*}}\right)+(1+c) \eta_{3} p^{*} G\left(\frac{p_{n}}{p^{*}}\right)\right] \\
= & s^{*}\left(\frac{s_{n+1}}{s^{*}}-\frac{s_{n}}{s^{*}}+\ln \frac{s_{n}}{s_{n+1}}\right)+\eta_{1} z^{*}\left(\frac{z_{n+1}}{z^{*}}-\frac{z_{n}}{z^{*}}+\ln \frac{z_{n}}{z_{n+1}}\right)+\eta_{2} u^{*}\left(\frac{u_{n+1}}{u^{*}}-\frac{u_{n}}{u^{*}}+\ln \frac{u_{n}}{u_{n+1}}\right) \\
+ & \eta_{3} p^{*}\left(\frac{p_{n+1}}{p^{*}}-\frac{p_{n}}{p^{*}}+\ln \frac{p_{n}}{p_{n+1}}\right)+c \eta_{3} p^{*}\left[G\left(\frac{p_{n+1}}{p^{*}}\right)-G\left(\frac{p_{n}}{p^{*}}\right)\right] .
\end{aligned}
$$

Using inequality (2.7), we get

$$
\begin{aligned}
\Delta u_{n} \leqslant & s^{*}\left(\frac{s_{n+1}-s_{n}}{s^{*}}+\frac{s_{n}}{s_{n+1}}-1\right)+\eta_{1} z^{*}\left(\frac{z_{n+1}-z_{n}}{z^{*}}+\frac{z_{n}}{z_{n+1}}-1\right)+\eta_{2} u^{*}\left(\frac{u_{n+1}-u_{n}}{u^{*}}+\frac{u_{n}}{u_{n+1}}-1\right) \\
& +\eta_{3} p^{*}\left(\frac{p_{n+1}-p_{n}}{p^{*}}+\frac{p_{n}}{p_{n+1}}-1\right)+c \eta_{3} p^{*}\left[G\left(\frac{p_{n+1}}{p^{*}}\right)-G\left(\frac{p_{n}}{p^{*}}\right)\right] \\
= & \left(1-\frac{s^{*}}{s_{n+1}}\right)\left(s_{n+1}-s_{n}\right)+\eta_{1}\left(1-\frac{z^{*}}{z_{n+1}}\right)\left(z_{n+1}-z_{n}\right)+\eta_{2}\left(1-\frac{u^{*}}{u_{n+1}}\right)\left(u_{n+1}-u_{n}\right) \\
& +\eta_{3}\left(1-\frac{p^{*}}{p_{n+1}}\right)\left(p_{n+1}-p_{n}\right)+c \eta_{3} p^{*}\left[G\left(\frac{p_{n+1}}{p^{*}}\right)-G\left(\frac{p_{n}}{p^{*}}\right)\right] .
\end{aligned}
$$

From Eqs. (2.1)-(2.4), we have

$$
\begin{aligned}
\Delta u_{n} \leqslant & \left(1-\frac{s^{*}}{s_{n+1}}\right)\left(\beta-\delta s_{n+1}-k s_{n+1} p_{n}\right)+\eta_{1}\left(1-\frac{z^{*}}{z_{n+1}}\right)\left((1-\alpha) k s_{n+1} p_{n}-d z_{n+1}\right) \\
& +\eta_{2}\left(1-\frac{u^{*}}{u_{n+1}}\right)\left(\alpha k s_{n+1} p_{n}-a u_{n+1}\right)+\eta_{3}\left(1-\frac{p^{*}}{p_{n+1}}\right)\left(N_{z} d z_{n+1}+N_{u} a u_{n+1}-c p_{n+1}\right) \\
& +c \eta_{3} p^{*}\left[G\left(\frac{p_{n+1}}{p^{*}}\right)-G\left(\frac{p_{n}}{p^{*}}\right)\right] .
\end{aligned}
$$

Since $\beta=\delta s^{*}+k s^{*} p^{*}$, then

$$
\Delta u_{n} \leqslant\left(1-\frac{s^{*}}{s_{n+1}}\right)\left(\delta s^{*}+k s^{*} p^{*}-\delta s_{n+1}-k s_{n+1} p_{n}\right)+\eta_{1}\left(1-\frac{z^{*}}{z_{n+1}}\right)\left((1-\alpha) k s_{n+1} p_{n}-d z_{n+1}\right)
$$




$$
\begin{aligned}
& +\eta_{2}\left(1-\frac{u^{*}}{u_{n+1}}\right)\left(\alpha k s_{n+1} p_{n}-a u_{n+1}\right)+\eta_{3}\left(1-\frac{p^{*}}{p_{n+1}}\right)\left(N_{z} d z_{n+1}+N_{u} a u_{n+1}-c p_{n+1}\right) \\
& +c \eta_{3} p^{*}\left[\frac{p_{n+1}}{p^{*}}-\frac{p_{n}}{p^{*}}+\ln \frac{p_{n}}{p_{n+1}}\right] \\
& =\left(1-\frac{s^{*}}{s_{n+1}}\right)\left(\delta s^{*}-\delta s_{n+1}\right)+\left(1-\frac{s^{*}}{s_{n+1}}\right) k s^{*} p^{*}+k s^{*} p_{n}-\eta_{1} \frac{z^{*}}{z_{n+1}}(1-\alpha) k s_{n+1} p_{n}+\eta_{1} d z^{*} \\
& -\eta_{2} \frac{u^{*}}{u_{n+1}} \alpha k s_{n+1} p_{n}+\eta_{2} a u^{*}-\eta_{3} \frac{p^{*}}{p_{n+1}}\left(N_{z} d z_{n+1}+N_{u} a u_{n+1}\right)+c \eta_{3} p^{*}+c \eta_{3} p^{*}\left(-\frac{p_{n}}{p^{*}}+\ln \frac{p_{n}}{p_{n+1}}\right) .
\end{aligned}
$$

Using the conditions of $\mathrm{Q}^{*}$

$$
(1-\alpha) k s^{*} p^{*}=d z^{*}, \quad \alpha k s^{*} p^{*}=a u^{*}, \quad N_{z} d z^{*}+N_{u} a u^{*}=c p^{*},
$$

we get

$$
k s^{*} p^{*}=\eta_{1} d z^{*}+\eta_{2} a u^{*}=\eta_{3} c p^{*},
$$

and

$$
\begin{aligned}
\Delta u_{n} \leqslant & \frac{-\delta}{s_{n+1}}\left(s_{n+1}-s^{*}\right)^{2}+\left(1-\frac{s^{*}}{s_{n+1}}\right)\left(\eta_{1} d z^{*}+\eta_{2} a u^{*}\right)-\eta_{1}(1-\alpha) k s^{*} p^{*} \frac{s_{n+1} p_{n} z^{*}}{s^{*} p^{*} z_{n+1}} \\
& +\eta_{1} d z^{*}-\eta_{2} \alpha k s^{*} p^{*} \frac{s_{n+1} p_{n} u^{*}}{s^{*} p^{*} u_{n+1}}+a \eta_{2} u^{*}-\eta_{3} N_{z} d z^{*} \frac{z_{n+1} p^{*}}{z^{*} p_{n+1}}-\eta_{3} N_{u} a u^{*} \frac{u_{n+1} p^{*}}{u^{*} p_{n+1}} \\
& +c \eta_{3} p^{*}+c \eta_{3} p^{*} \ln \frac{p_{n}}{p_{n+1}} \\
= & \frac{-\delta}{s_{n+1}}\left(s_{n+1}-s^{*}\right)^{2}+\left(1-\frac{s^{*}}{s_{n+1}}\right)\left(\eta_{1} d z^{*}+\eta_{2} a u^{*}\right)-\eta_{1} d z^{*} \frac{s_{n+1} p_{n} z^{*}}{s^{*} p^{*} z_{n+1}}+\eta_{1} d z^{*} \\
& -\eta_{2} a u^{*} \frac{s_{n+1} p_{n} u^{*}}{s^{*} p^{*} u_{n+1}}+a \eta_{2} u^{*}-\eta_{1} d z^{*} \frac{z_{n+1} p^{*}}{z^{*} p_{n+1}}-\eta_{2} a u^{*} \frac{u_{n+1} p^{*}}{u^{*} p_{n+1}}+\eta_{1} d z^{*}+\eta_{2} a u^{*} \\
& +\left(\eta_{1} d z^{*}+\eta_{2} a u^{*}\right) \ln \frac{p_{n}}{p_{n+1}} \\
= & \frac{-\delta}{s_{n+1}}\left(s_{n+1}-s^{*}\right)^{2}+\eta_{1} d z^{*}\left(3-\frac{s^{*}}{s_{n+1}}-\frac{s_{n+1} p_{n} z^{*}}{s^{*} p^{*} z_{n+1}}-\frac{z_{n+1} p^{*}}{z^{*} p_{n+1}}+\ln \frac{p_{n}}{p_{n+1}}\right) \\
& +\eta_{2} a u^{*}\left(3-\frac{s^{*}}{s_{n+1}}-\frac{s_{n+1} p_{n} u^{*}}{s^{*} p^{*} u_{n+1}}-\frac{u_{n+1} p^{*}}{u^{*} p_{n+1}}+\ln \frac{p_{n}}{p_{n+1}}\right), \\
\Delta u_{n} \leqslant & \frac{-\delta}{s_{n+1}}\left(s_{n+1}-s^{*}\right)^{2}-\eta_{1} d z^{*}\left[G\left(\frac{s^{*}}{s_{n+1}}\right)+G\left(\frac{s_{n+1} p_{n} z^{*}}{s^{*} p^{*} z_{n+1}}\right)+G\left(\frac{z_{n+1} p^{*}}{z^{*} p_{n+1}}\right)\right] \\
& -\eta_{2} a u^{*}\left[G\left(\frac{s^{*}}{s_{n+1}}\right)+G\left(\frac{s_{n+1} p_{n} u^{*}}{s_{*}^{*} p^{*} u_{n+1}}\right)+G\left(\frac{u_{n+1} p^{*}}{u^{*} p_{n+1}}\right)\right] .
\end{aligned}
$$

Thus, $u_{n}$ is monotone decreasing sequence. Because $u_{n} \geqslant 0$, there is a limit $\lim _{n \rightarrow \infty} u_{n} \geqslant 0$. Therefore, $\lim _{n \rightarrow \infty} \Delta U_{n}=0$, which implies $\lim _{n \rightarrow \infty} s_{n}=s^{*}, \lim _{n \rightarrow \infty} z_{n}=z^{*}, \lim _{n \rightarrow \infty} u_{n}=u^{*}$ and $\lim _{n \rightarrow \infty} p_{n}=p^{*}$.

\section{Model with saturated incidence}

It has been reported in $[37,38]$ that HIV dynamics model with saturated incidence is more accurate in case of high concentration of the HIV particles. Thus we consider the following model:

$$
\dot{\mathrm{s}}=\beta-\delta \mathrm{s}-\frac{\mathrm{ksp}}{1+\mu \mathrm{p}}, \quad \dot{z}=\frac{(1-\alpha) k s p}{1+\mu \mathrm{p}}-\mathrm{d} z, \quad \dot{\mathrm{u}}=\frac{\alpha \mathrm{ksp}}{1+\mu \mathrm{p}}-\mathrm{au}, \quad \dot{\mathrm{p}}=\mathrm{N}_{z} \mathrm{~d} z+\mathrm{N}_{\mathrm{u}} \mathrm{au}-\mathrm{cp},
$$

where $\mu$ is the saturation constant. Using the NSFD method we obtain

$$
s_{n+1}-s_{n}=\beta-\delta s_{n+1}-\frac{k s_{n+1} p_{n}}{1+\mu p_{n}}
$$




$$
\begin{aligned}
& z_{n+1}-z_{n}=\frac{(1-\alpha) k s_{n+1} p_{n}}{1+\mu p_{n}}-d z_{n+1} \\
& u_{n+1}-u_{n}=\frac{\alpha k s_{n+1} p_{n}}{1+\mu p_{n}}-a u_{n+1} \\
& p_{n+1}-p_{n}=N_{z} d z_{n+1}+N_{u} a u_{n+1}-c p_{n+1}
\end{aligned}
$$

Now we study the basic and global properties of model (3.1)-(3.4).

\subsection{Preliminaries}

Lemma 3.1. Any solution $\left(s_{n}, z_{n}, u_{n}, p_{n}\right)$ of model (3.1)-(3.4) with initial conditions (2.5) is positive and ultimately bounded.

Proof. From Eqs. (3.1)-(3.4) we obtain

$$
\begin{aligned}
s_{n+1}= & \frac{\left(\beta+s_{n}\right)\left(1+\mu p_{n}\right)}{1+\delta+(\mu(1+\delta)+k) p_{n}}, \\
z_{n+1}= & \frac{z_{n}}{1+d}+\frac{(1-\alpha) k p_{n}\left(\beta+s_{n}\right)}{(1+d)\left(1+\delta+(\mu(1+\delta)+k) p_{n}\right)}, \\
u_{n+1}= & \frac{u_{n}}{1+a}+\frac{\alpha k p_{n}\left(\beta+s_{n}\right)}{(1+a)\left(1+\delta+(\mu(1+\delta)+k) p_{n}\right)^{\prime}}, \\
p_{n+1}= & \frac{p_{n}}{1+c}+\frac{N_{z} d}{1+c}\left(\frac{z_{n}}{1+d}+\frac{(1-\alpha) k p_{n}\left(\beta+s_{n}\right)}{(1+d)\left(1+\delta+(\mu(1+\delta)+k) p_{n}\right)}\right) \\
& +\frac{N_{c} a}{1+c}\left(\frac{u_{n}}{1+a}+\frac{\alpha k p_{n}\left(\beta+s_{n}\right)}{(1+a)\left(1+\delta+(\mu(1+\delta)+k) p_{n}\right)}\right) .
\end{aligned}
$$

The solution of (3.1)-(3.4) with initial (2.5) satisfies $s_{n}>0, z_{n}>0, u_{n}>0$ and $p_{n}>0$. The boundedness of solutions of model (3.1)-(3.4) is similar to the proof of Lemma 2.1.

System (3.1)-(3.4) has two equilibria,

(i) HIV-free equilibrium $\mathrm{Q}^{0}\left(s^{0}, 0,0,0\right)$ where $s^{0}=\beta / \delta$;

(ii) persistent HIV equilibrium $\mathrm{Q}^{*}\left(\mathrm{~s}^{*}, z^{*}, \mathrm{u}^{*}, \mathrm{p}^{*}\right)$, where

$$
\begin{aligned}
s^{*} & =\frac{\mu \beta\left[N_{z}(1-\alpha)+\alpha N_{u}\right]+c}{(k+\delta \mu)\left[N_{z}(1-\alpha)+\alpha N_{u}\right]}, & z^{*} & =\frac{(1-\alpha) c \delta}{d(k+\delta \mu)\left[N_{z}(1-\alpha)+\alpha N_{u}\right]}\left(\mathcal{R}_{0}-1\right), \\
u^{*} & =\frac{\alpha c \delta}{a(k+\delta \mu)\left[N_{z}(1-\alpha)+\alpha N_{u}\right]}\left(\mathcal{R}_{0}-1\right), & p^{*} & =\frac{\delta}{k+\delta \mu}\left(\mathcal{R}_{0}-1\right),
\end{aligned}
$$

where $\mathcal{R}_{0}$ is given by Eq. (2.6).

\subsection{Global Stability}

Theorem 3.2. If $\mathcal{R}_{0} \leqslant 1$, then $\mathrm{Q}^{0}$ is globally asymptotically stable.

Proof. Construct a discrete Lyapunov function $L_{n}\left(s_{n}, z_{n}, u_{n}, p_{n}\right)$ as:

$$
L_{n}=s^{0} G\left(\frac{s_{n}}{s^{0}}\right)+\eta_{1} z_{n}+\eta_{2} u_{n}+\eta_{3}(1+c) p_{n},
$$

where $\eta_{i}, i=1,2,3$ are given by Eq. (2.9). Hence, $L_{n} \geqslant 0$ for all $s_{n}>0, z_{n}>0, u_{n}>0$ and $p_{n}>0$. In addition, $L_{n}=0$ if and only if $s_{n}=s^{0}, z_{n}=0, u_{n}=0$ and $p_{n}=0$. Computing the difference $\Delta \mathrm{L}_{\mathrm{n}}=\mathrm{L}_{\mathrm{n}+1}-\mathrm{L}_{\mathrm{n}}$ as:

$$
\Delta L_{n}=s^{0} G\left(\frac{s_{n+1}}{s^{0}}\right)+\eta_{1} z_{n+1}+\eta_{2} u_{n+1}+\eta_{3}(1+c) p_{n+1}-\left[s^{0} G\left(\frac{s_{n}}{s^{0}}\right)+\eta_{1} z_{n}+\eta_{2} u_{n}+\eta_{3}(1+c) p_{n}\right]
$$




$$
=s^{0}\left(\frac{s_{n+1}}{s^{0}}-\frac{s_{n}}{s^{0}}+\ln \frac{s_{n}}{s_{+1}}\right)+\eta_{1}\left(z_{n+1}-z_{n}\right)+\eta_{2}\left(u_{n+1}-u_{n}\right)+\eta_{3}(1+c)\left(p_{n+1}-p_{n}\right) .
$$

Using inequality (2.7), we have

$$
\begin{aligned}
\Delta L_{n} & \leqslant s_{n+1}-s_{n}+s^{0}\left(\frac{s_{n}}{s_{n+1}}-1\right)+\eta_{1}\left(z_{n+1}-z_{n}\right)+\eta_{2}\left(u_{n+1}-u_{n}\right)+\eta_{3}(1+c)\left(p_{n+1}-p_{n}\right) \\
& =\left(1-\frac{s^{0}}{s_{n+1}}\right)\left(s_{n+1}-s_{n}\right)+\eta_{1}\left(z_{n+1}-z_{n}\right)+\eta_{2}\left(u_{n+1}-u_{n}\right)+\eta_{3}(1+c)\left(p_{n+1}-p_{n}\right) .
\end{aligned}
$$

From Eqs. (3.1)-(3.4), we have

$$
\begin{aligned}
\Delta \mathrm{L}_{n} \leqslant & \left(1-\frac{s^{0}}{s_{n+1}}\right)\left(\beta-\delta s_{n+1}-\frac{k s_{n+1} p_{n}}{1+\mu p_{n}}\right)+\eta_{1}\left(\frac{(1-\alpha) k s_{n+1} p_{n}}{1+\mu p_{n}}-d z_{n+1}\right) \\
& +\eta_{2}\left(\frac{\alpha k s_{n+1} p_{n}}{1+\mu p_{n}}-a u_{n+1}\right)+\eta_{3}\left(N_{z} d z_{n+1}+N_{u} a u_{n+1}-c p_{n+1}\right)+\eta_{3} c\left(p_{n+1}-p_{n}\right) \\
= & \left(1-\frac{s^{0}}{s_{n+1}}\right)\left(\beta-\delta s_{n+1}\right)+\frac{k s_{0} p_{n}}{1+\mu p_{n}}-\eta_{3} c p_{n} \\
= & \frac{-\delta}{s_{n+1}}\left(s_{n+1}-s^{0}\right)^{2}+\left(\frac{k s_{0} p_{n}}{1+\mu p_{n}}-\frac{c}{N_{z}(1-\alpha)+\alpha N_{u}}\right) p_{n} \\
= & \frac{-\delta}{s_{n+1}}\left(s_{n+1}-s^{0}\right)^{2}+\frac{c}{N_{z}(1-\alpha)+\alpha N_{u}}\left(\frac{\left(N_{z}(1-\alpha)+\alpha N_{u}\right) k s_{0}}{c\left(1+\mu p_{n}\right)}-1\right) p_{n} \\
= & \frac{-\delta}{s_{n+1}}\left(s_{n+1}-s^{0}\right)^{2}+\eta_{3} c\left(\frac{\mathcal{R}_{0}}{1+\mu p_{n}}-1\right) p_{n} \\
= & \frac{-\delta}{s_{n+1}}\left(s_{n+1}-s^{0}\right)^{2}+\eta_{3} c\left(\mathcal{R}_{0}-\frac{\mathcal{R}_{0} \mu p_{n}}{1+\mu p_{n}}-1\right) p_{n} \\
= & \frac{-\delta}{s_{n+1}}\left(s_{n+1}-s^{0}\right)^{2}-\eta_{3} c \frac{\mathcal{R}_{0} \mu p_{n}^{2}}{1+\mu p_{n}}+\eta_{3} c\left(\mathcal{R}_{0}-1\right) p_{n} .
\end{aligned}
$$

Hence, for $\mathcal{R}_{0} \leqslant 1$, we have $\Delta \mathrm{L}_{n} \leqslant 0$ for all $\mathrm{n} \geqslant 0$. This yields that $\mathrm{L}_{n}$ is a monotone decreasing sequence. The proof can be completed similar to that of Theorem 2.2.

Theorem 3.3. If $\mathcal{R}_{0}>1$, then $\mathrm{Q}^{*}$ is globally asymptotically stable.

Proof. Let us consider

$$
u_{n}\left(s_{n}, z_{n}, u_{n}, p_{n}\right)=s^{*} G\left(\frac{s_{n}}{s^{*}}\right)+\eta_{1} z^{*} G\left(\frac{z_{n}}{z^{*}}\right)+\eta_{2} u^{*} G\left(\frac{u_{n}}{u^{*}}\right)+\eta_{3}(1+c) p^{*} G\left(\frac{p_{n}}{p^{*}}\right)
$$

Clearly, $u_{n}\left(s_{n}, z_{n}, u_{n}, p_{n}\right)>0$ for all $s_{n}, z_{n}, u_{n}, p_{n}>0$ and $u_{n}\left(s^{*}, z^{*}, u^{*}, p^{*}\right)=0$. Computing $\Delta u_{n}=$ $\mathrm{U}_{\mathrm{n}+1}-\mathrm{U}_{\mathrm{n}}$ as:

$$
\begin{aligned}
\Delta u_{n}= & s^{*} G\left(\frac{s_{n+1}}{s^{*}}\right)+\eta_{1} z^{*} G\left(\frac{z_{n+1}}{z^{*}}\right)+\eta_{2} u^{*} G\left(\frac{u_{n+1}}{u^{*}}\right)+\eta_{3}(1+c) p^{*} G\left(\frac{p_{n+1}}{p^{*}}\right) \\
& -\left[s^{*} G\left(\frac{s_{n}}{s^{*}}\right)+\eta_{1} z^{*} G\left(\frac{z_{n}}{z^{*}}\right)+\eta_{2} u^{*} G\left(\frac{u_{n}}{u^{*}}\right)+\eta_{3}(1+c) p^{*} G\left(\frac{p_{n}}{p^{*}}\right)\right] \\
= & s^{*}\left(\frac{s_{n+1}}{s^{*}}-\frac{s_{n}}{s^{*}}+\ln \frac{s_{n}}{s_{n+1}}\right)+\eta_{1} z^{*}\left(\frac{z_{n+1}}{z^{*}}-\frac{z_{n}}{z^{*}}+\ln \frac{z_{n}}{z_{n+1}}\right)+\eta_{2} u^{*}\left(\frac{u_{n+1}}{u^{*}}-\frac{u_{n}}{u^{*}}+\ln \frac{u_{n}}{u_{n+1}}\right) \\
& +\eta_{3} p^{*}\left(\frac{p_{n+1}}{p^{*}}-\frac{p_{n}}{p^{*}}+\ln \frac{p_{n}}{p_{n+1}}\right)+\eta_{3} c p^{*}\left[G\left(\frac{p_{n+1}}{p^{*}}\right)-G\left(\frac{p_{n}}{p^{*}}\right)\right] .
\end{aligned}
$$


Using inequality (2.7), we get

$$
\begin{aligned}
\Delta u_{n} \leqslant & s^{*}\left(\frac{s_{n+1}-s_{n}}{s^{*}}+\frac{s_{n}}{s_{n+1}}-1\right)+\eta_{1} z^{*}\left(\frac{z_{n+1}-z_{n}}{z^{*}}+\frac{z_{n}}{z_{n+1}}-1\right)+\eta_{2} u^{*}\left(\frac{u_{n+1}-u_{n}}{u^{*}}+\frac{u_{n}}{u_{n+1}}-1\right) \\
& +\eta_{3} p^{*}\left(\frac{p_{n+1}-p_{n}}{p^{*}}+\frac{p_{n}}{p_{n+1}}-1\right)+\eta_{3} c p^{*}\left[G\left(\frac{p_{n+1}}{p^{*}}\right)-G\left(\frac{p_{n}}{p^{*}}\right)\right] \\
= & \left(1-\frac{s^{*}}{s_{n+1}}\right)\left(s_{n+1}-s_{n}\right)+\eta_{1}\left(1-\frac{z^{*}}{z_{n+1}}\right)\left(z_{n+1}-z_{n}\right)+\eta_{2}\left(1-\frac{u^{*}}{u_{n+1}}\right)\left(u_{n+1}-u_{n}\right) \\
& +\eta_{3}\left(1-\frac{p^{*}}{p_{n+1}}\right)\left(p_{n+1}-p_{n}\right)+\eta_{3} c p^{*}\left[G\left(\frac{p_{n+1}}{p^{*}}\right)-G\left(\frac{p_{n}}{p^{*}}\right)\right] .
\end{aligned}
$$

From Eqs. (3.1)-(3.4), we have

$$
\begin{aligned}
\Delta \mathrm{u}_{\mathrm{n}} \leqslant & \left(1-\frac{s^{*}}{s_{n+1}}\right)\left(\beta-\delta s_{n+1}-\frac{k s_{n+1} p_{n}}{1+\mu p_{n}}\right)+\eta_{1}\left(1-\frac{z^{*}}{z_{n+1}}\right)\left(\frac{(1-\alpha) k s_{n+1} p_{n}}{1+\mu p_{n}}-d z_{n+1}\right) \\
& +\eta_{2}\left(1-\frac{u^{*}}{u_{n+1}}\right)\left(\frac{\alpha k s_{n+1} p_{n}}{1+\mu p_{n}}-a u_{n+1}\right)+\eta_{3}\left(1-\frac{p^{*}}{p_{n+1}}\right)\left(N_{z} d z_{n+1}+N_{u} a u_{n+1}-c p_{n+1}\right) \\
& +\eta_{3} c p^{*}\left(\frac{p_{n+1}}{p^{*}}-\frac{p_{n}}{p^{*}}+\ln \frac{p_{n}}{p_{n+1}}\right) \\
= & \left(1-\frac{s^{*}}{s_{n+1}}\right)\left(\beta-\delta s_{n+1}\right)+\frac{k s^{*} p_{n}}{1+\mu p_{n}}-\eta_{1} \frac{z^{*}}{z_{n+1}} \frac{(1-\alpha) k s_{n+1} p_{n}}{1+\mu p_{n}}+\eta_{1} d z^{*} \\
& -\eta_{2} \frac{u^{*}}{u_{n+1}} \frac{\alpha k s_{n+1} p_{n}}{1+\mu p_{n}}+\eta_{2} a u^{*}-\eta_{3} \frac{p^{*}}{p_{n+1}}\left(N_{z} d z_{n+1}+N_{u} a u_{n+1}\right)+\eta_{3} c p^{*}+\eta_{3} c p^{*}\left(-\frac{p_{n}}{p^{*}}+\ln \frac{p_{n}}{p_{n+1}}\right) .
\end{aligned}
$$

Using the conditions of $\mathrm{Q}^{*}$

$$
\beta=\delta s^{*}+\frac{k s^{*} p^{*}}{1+\mu p^{*}}, \quad \frac{(1-\alpha) k s^{*} p^{*}}{1+\mu p^{*}}=d z^{*}, \quad \frac{\alpha k s^{*} p^{*}}{1+\mu p^{*}}=a u^{*}, \quad c p^{*}=N_{z} d z^{*}+N_{u} a u^{*} ，
$$

we get

$$
\frac{k s^{*} p^{*}}{1+\mu p^{*}}=\eta_{1} d z^{*}+\eta_{2} a u^{*}=\eta_{3} c p^{*}
$$

and

$$
\begin{aligned}
\Delta u_{n} \leqslant & \left(1-\frac{s^{*}}{s_{n+1}}\right)\left(\delta s^{*}+\frac{k s^{*} p^{*}}{1+\mu p^{*}}-\delta s_{n+1}\right)+\frac{k s^{*} p_{n}}{1+\mu p_{n}}-\eta_{1} \frac{(1-\alpha) k s^{*} p^{*}}{1+\mu p^{*}} \frac{s_{n+1} p_{n} z^{*}\left(1+\mu p^{*}\right)}{s^{*} p^{*} z_{n+1}\left(1+\mu p_{n}\right)} \\
& +\eta_{1} d z^{*}-\eta_{2} \frac{\alpha k s^{*} p^{*}}{1+\mu p^{*}} \frac{s_{n+1} p_{n} u^{*}\left(1+\mu p^{*}\right)}{s^{*} p^{*} u_{n+1}\left(1+\mu p_{n}\right)}+\eta_{2} a u^{*}-\eta_{1} d z^{*} \frac{p^{*} z_{n+1}}{p_{n+1} z^{*}}-\eta_{2} a u^{*} \frac{p^{*} u_{n+1}}{p_{n+1} u^{*}}+\eta_{3} c p^{*} \\
& +\eta_{3} c p^{*}\left(-\frac{p_{n}}{p^{*}}+\ln \frac{p_{n}}{p_{n+1}}\right) \\
= & \left(1-\frac{s^{*}}{s_{n+1}}\right)\left(\delta s^{*}-\delta s_{n+1}\right)+\frac{k s^{*} p^{*}}{1+\mu p^{*}}\left(1-\frac{s^{*}}{s_{n+1}}\right)+\frac{k s^{*} p^{*}}{1+\mu p^{*}} \frac{p_{n}\left(1+\mu p^{*}\right)}{p^{*}\left(1+\mu p_{n}\right)} \\
& -\eta_{1} d z^{*} \frac{s_{n+1} p_{n} z^{*}\left(1+\mu p^{*}\right)}{s^{*} p^{*} z_{n+1}\left(1+\mu p_{n}\right)}+\eta_{1} d z^{*}-\eta_{2} a u^{*} \frac{s_{n+1} p_{n} u^{*}\left(1+\mu p^{*}\right)}{s^{*} p^{*} u_{n+1}\left(1+\mu p_{n}\right)}+\eta_{2} a u^{*}-\eta_{1} d z^{*} \frac{z_{n+1} p^{*}}{z^{*} p_{n+1}} \\
& -\eta_{2} a u^{*} \frac{u_{n+1} p^{*}}{u^{*} p_{n+1}}+\eta_{1} d z^{*}+\eta_{2} a u^{*}+\frac{k s^{*} p^{*}}{1+\mu p^{*}}\left(-\frac{p_{n}}{p^{*}}+\ln \frac{p_{n}}{p_{n+1}}\right) \\
= & \frac{-\delta}{s_{n+1}}\left(s_{n+1}-s^{*}\right)^{2}+\frac{k s^{*} p^{*}}{1+\mu p^{*}}\left(-1+\frac{p_{n}\left(1+\mu p^{*}\right)}{p^{*}\left(1+\mu p_{n}\right)}-\frac{p_{n}}{p *}+\frac{1+\mu p_{n}}{1+\mu p^{*}}\right) \\
& +\eta_{1} d z^{*}\left[4-\frac{s^{*}}{s_{n+1}}-\frac{s_{n+1} p_{n} z^{*}\left(1+\mu p^{*}\right)}{s^{*} p^{*} z_{n+1}\left(1+\mu p_{n}\right)}-\frac{z_{n+1} p^{*}}{z^{*} p_{n+1}}-\frac{1+\mu p_{n}}{1+\mu p^{*}}+\ln \frac{p_{n}}{p_{n+1}}\right]
\end{aligned}
$$




$$
+\eta_{2} a u^{*}\left[4-\frac{s^{*}}{s_{n+1}}-\frac{s_{n+1} p_{n} u^{*}\left(1+\mu p^{*}\right)}{s^{*} p^{*} u_{n+1}\left(1+\mu p_{n}\right)}-\frac{u_{n+1} p^{*}}{u^{*} p_{n+1}}-\frac{1+\mu p_{n}}{1+\mu p^{*}}+\ln \frac{p_{n}}{p_{n+1}}\right]
$$

We have

$$
-1+\frac{p_{n}\left(1+\mu p^{*}\right)}{p^{*}\left(1+\mu p_{n}\right)}-\frac{p_{n}}{p^{*}}+\frac{1+\mu p_{n}}{1+\mu p^{*}}=-\frac{\mu\left(p_{n}-p^{*}\right)^{2}}{p^{*}\left(1+\mu p^{*}\right)\left(1+\mu p_{n}\right)}
$$

Then

$$
\begin{aligned}
\Delta u_{n} \leqslant & \frac{-\delta}{s_{n+1}}\left(s_{n+1}-s^{*}\right)^{2}-\frac{k s^{*} p^{*}}{1+\mu p^{*}}\left(\frac{\mu\left(p_{n}-p^{*}\right)^{2}}{p^{*}\left(1+\mu p^{*}\right)\left(1+\mu p_{n}\right)}\right) \\
& -\eta_{1} d z^{*}\left[G\left(\frac{s^{*}}{s_{n+1}}\right)+G\left(\frac{s_{n+1} p_{n} z^{*}}{s^{*} p^{*} z_{n+1}} \frac{\left(1+\mu p^{*}\right)}{\left(1+\mu p_{n}\right)}\right)+G\left(\frac{z_{n+1} p^{*}}{z^{*} p_{n+1}}\right)+G\left(\frac{1+\mu p_{n}}{1+\mu p^{*}}\right)\right] \\
& -\eta_{2} a u^{*}\left[G\left(\frac{s^{*}}{s_{n+1}}\right)+G\left(\frac{s_{n+1} p_{n} u^{*}}{s^{*} p^{*} u_{n+1}} \frac{\left(1+\mu p^{*}\right)}{\left(1+\mu p_{n}\right)}\right)+G\left(\frac{u_{n+1} p^{*}}{u^{*} p_{n+1}}\right)+G\left(\frac{1+\mu p_{n}}{1+\mu p^{*}}\right)\right] .
\end{aligned}
$$

Thus, $U_{n}$ is monotone decreasing sequence. Because $U_{n} \geqslant 0$, there is a limit $\lim _{n \rightarrow \infty} U_{n} \geqslant 0$, and hence, $\lim _{n \rightarrow \infty} \Delta u_{n}=0$, which implies that $\lim _{n \rightarrow \infty} s_{n}=s^{*}, \lim _{n \rightarrow \infty} z_{n}=z^{*}, \lim _{n \rightarrow \infty} u_{n}=u^{*}$ and $\lim _{n \rightarrow \infty} p_{n}=p^{*}$.

\section{Model with general incidence}

In this section, we assume that the incidence rate is given by $f(s, p)$, where $f$ is a general function.

$$
\dot{\mathrm{s}}=\beta-\delta s-f(s, p), \quad \dot{z}=(1-\alpha) f(s, p)-d z, \quad \dot{u}=\alpha f(s, p)-a u, \quad \dot{p}=N_{z} d z+N_{u} a u-c p .
$$

Using the NSFD method we get

$$
\begin{aligned}
s_{n+1}-s_{n} & =\beta-\delta s_{n+1}-f\left(s_{n+1}, p_{n}\right) \\
z_{n+1}-z_{n} & =(1-\alpha) f\left(s_{n+1}, p_{n}\right)-d z_{n+1} \\
u_{n+1}-u_{n} & =\alpha f\left(s_{n+1}, p_{n}\right)-a u_{n+1} \\
p_{n+1}-p_{n} & =N_{z} d z_{n+1}+N_{u} a u_{n+1}-c p_{n+1} .
\end{aligned}
$$

\subsection{Preliminaries}

The function $f(s, p)$ is assumed to satisfy the following conditions:

(A1) $f(s, p)>0$, and $f(0, p)=f(s, 0)=0$ for all $s>0, p>0$;

(A2) $\frac{\partial f(s, p)}{\partial s}>0, \frac{\partial f(s, p)}{\partial p}>0, \frac{\partial f(s, 0)}{\partial p}>0$ for all $s>0, p>0$;

(A3) $\frac{d}{d s}\left(\frac{\partial f(s, 0)}{\partial p}\right)>0$ for all $s>0$;

(A4) $\frac{f(s, p)}{p}$ is decreasing with respect to $p$ for all $p>0$.

Lemma 4.1. Any solution $\left(s_{n}, z_{n}, u_{n}, p_{n}\right)$ of model (4.1)-(4.4) with initial conditions (2.5) is positive and ultimately bounded.

Proof. From Eqs. (4.1)-(4.4) we obtain

$$
\begin{aligned}
& s_{n+1}=\frac{s_{n}+\beta-f\left(s_{n+1}, p_{n}\right)}{1+\delta}, \\
& z_{n+1}=\frac{z_{n}+(1-\alpha) f\left(s_{n+1}, p_{n}\right)}{1+d}, \\
& u_{n+1}=\frac{u_{n}+\alpha f\left(s_{n+1}, p_{n}\right)}{1+a},
\end{aligned}
$$




$$
p_{n+1}=\frac{p_{n}+N_{z} d z_{n+1}+N_{u} a u_{n+1}}{1+c}
$$

When $\mathrm{n}=0$ we prove that $\left(\mathrm{s}_{1}, z_{1}, \mathrm{u}_{1}, \mathrm{p}_{1}\right)$ exists and is positive. From Eq. (4.1) we have

$$
(1+\delta) s_{1}-s_{0}-\beta+f\left(s_{1}, p_{0}\right)=0 .
$$

Let $\varphi$ be defined as:

$$
\varphi\left(s_{1}\right)=(1+\delta) s_{1}-s_{0}-\beta+f\left(s_{1}, p_{0}\right)=0, \quad \varphi(0)=-s_{0}-\beta<0, \quad \lim _{s_{1} \rightarrow \infty} \varphi\left(s_{1}\right)=\infty .
$$

From Assumption (A2), $\varphi$ is a strictly increasing function in $s_{1}$. Hence, there exists a unique $s_{1}>0$ such that $\varphi\left(s_{1}\right)=0$. From Eqs. (4.5)-(4.7) we have $z_{1}>0, u_{1}>0$ and $p_{1}>0$. Therefore, by using the induction, we obtain $s_{n}>0, z_{n}>0, u_{n}>0$ and $p_{n}>0$ for all $n \geqslant 0$. The boundedness of solutions can be shown similar to Lemma 2.1.

Lemma 4.2. For model (4.1)-(4.4) let (A1)-(A2) hold true, then there exists a threshold parameter $\mathcal{R}_{0}>0$ such that

(i) if $\mathcal{R}_{0} \leqslant 1$, then there exists only an HIV-free equilibrium $\mathrm{Q}^{0}$;

(ii) if $\mathcal{R}_{0}>1$, then there exist two equilibria, $\mathrm{Q}^{0}$ and a persistent HIV equilibrium $\mathrm{Q}^{*}$.

Proof. Let $\mathrm{Q}(s, z, \mathrm{u}, \mathrm{p})$ be any equilibrium of model (4.1)-(4.4) satisfying

$$
\begin{aligned}
\beta-\delta s-f(s, p) & =0, \\
(1-\alpha) f(s, p)-d z & =0, \\
\alpha f(s, p)-a u & =0, \\
N_{z} d z+N_{u} a u-c p & =0 .
\end{aligned}
$$

From Eqs. (4.8)-(4.10) we have

$$
z=\frac{(1-\alpha) f(s, p)}{d}, \quad \quad u=\frac{\alpha f(s, p)}{a}, \quad \quad f(s, p)=\beta-\delta s .
$$

Substituting from Eq. (4.12) into Eq. (4.11) we get

$$
\left(N_{z}(1-\alpha)+N_{u} \alpha\right) f(s, p)-c p=0 .
$$

From Assumption (A1), we have $p=0$ is a solution of (4.13). Therefore, $s=s^{0}, z=0$ and $u=0$ which leads to the HIV-free equilibrium $Q^{0}\left(s^{0}, 0,0,0\right)$ where $s^{0}=\frac{\beta}{\delta}$. If $p \neq 0$, then, from Eqs. (4.12), (4.13), we obtain

$$
p=\frac{\gamma f(s, p)}{c}=\frac{\gamma(\beta-\delta s)}{c}, \quad s=s^{0}-\frac{p c}{\gamma \delta}
$$

where, $\gamma=\mathrm{N}_{z}(1-\alpha)+\mathrm{N}_{u} \alpha$. Then, Eq. (4.13) becomes

$$
\gamma f\left(s^{0}-\frac{c}{\gamma \delta} p, p\right)-c p=0 .
$$

Let a function $\psi_{1}$ be defined as:

$$
\psi_{1}(p)=\gamma f\left(s^{0}-\frac{c}{\gamma \delta} p, p\right)-c p=0 .
$$

From Assumption (A1), we have $\psi_{1}(0)=0$, and $\psi_{1}(\bar{p})=-c \bar{p}<0$, where $\bar{p}=\frac{\gamma \beta}{c}>0$. Moreover,

$$
\psi_{1}^{\prime}(0)=\gamma \frac{\partial f\left(s^{0}, 0\right)}{\partial p}-c=c\left(\frac{\gamma}{c} \frac{\partial f\left(s^{0}, 0\right)}{\partial p}-1\right) .
$$


Therefore, $\psi^{\prime}(0)>0$ if the following condition is satisfied

$$
\frac{\gamma}{c} \frac{\partial f\left(s^{0}, 0\right)}{\partial p}>1
$$

It follows that, if condition (4.14) is satisfied, then there exists $p^{*} \in(0, \bar{p})$ such that $\psi\left(p^{*}\right)=0$. Hence, we can define the basic reproduction number of system (4.1)-(4.4) as:

$$
\mathcal{R}_{0}=\frac{\gamma}{\mathrm{c}} \frac{\partial f\left(\mathrm{~s}^{0}, 0\right)}{\partial \mathrm{p}}
$$

Moreover, let $p=p^{*}$ in Eq. (4.8) we get

$$
\beta-\delta s-f\left(s, p^{*}\right)=0
$$

Let us define

$$
\psi_{2}(s)=\beta-\delta s-f\left(s, p^{*}\right) .
$$

We have $\psi_{2}(0)=\beta>0$ and $\psi_{2}\left(s^{0}\right)=-f\left(s^{0}, p^{*}\right)<0$. Since $f(s, p)$ is strictly decreasing with respect to $s$, then $\psi_{1}(s)$ is strictly decreasing with respect to $s$. Hence, there exists a unique $s^{*} \in\left(0, s^{0}\right)$ such that $\psi_{1}\left(s^{*}\right)=0$. From Eq. (4.12) and Assumption (A1) we have

$$
z^{*}=\frac{(1-\alpha) f\left(s^{*}, p^{*}\right)}{d}>0, \quad u^{*}=\frac{\alpha f\left(s^{*}, p^{*}\right)}{a}>0 .
$$

This shows that if $\mathcal{R}_{0}>1$, then there exists a persistent-HIV equilibrium $\mathrm{Q}^{*}\left(\mathrm{~s}^{*}, w^{*}, \mathrm{u}^{*}, \mathrm{p}^{*}\right)$.

\subsection{Global stability}

Theorem 4.3. Suppose that $\mathcal{R}_{0} \leqslant 1$, then $\mathrm{Q}^{0}$ of system (4.1)-(4.4) is globally asymptotically stable.

Proof. Define

$$
L_{n}=s_{n}-s^{0}-\int_{s^{0}}^{s_{n}} \lim _{p \rightarrow 0^{+}} \frac{f\left(s^{0}, p\right)}{f(\tau, p)} d \tau+\eta_{1} z_{n}+\eta_{2} u_{n}+\eta_{3}(1+c) p_{n}
$$

Hence, $L_{n}>0$ for all $s_{n}, z_{n}, u_{n}, p_{n}>0$ and $L_{n}=0$ if and only if $s_{n}=s^{0}, z_{n}=0, u_{n}=0$ and $p_{n}=0$. Computing the difference $\Delta \mathrm{L}_{n}=\mathrm{L}_{n+1}-\mathrm{L}_{n}$ as:

$$
\begin{aligned}
\Delta L_{n}= & s_{n+1}-s^{0}-\int_{s^{0}}^{s_{n+1}} \lim _{p \rightarrow 0^{+}} \frac{f\left(s^{0}, p\right)}{f(\tau, p)} d \tau+\eta_{1} z_{n+1}+\eta_{2} u_{n+1}+\eta_{3}(1+c) p_{n+1} \\
& -\left[s_{n}-s^{0}-\int_{s^{0}}^{s_{n}} \lim _{p \rightarrow 0^{+}} \frac{f\left(s^{0}, p\right)}{f(\tau, p)} d \tau+\eta_{1} z_{n}+\eta_{2} u_{n}+\eta_{3}(1+c) p_{n}\right] \\
= & s_{n+1}-s_{n}-\int_{s_{n}}^{s_{n+1}} \lim _{p \rightarrow 0^{+}} \frac{f\left(s^{0}, p\right)}{f(\tau, p)} d \tau+\eta_{1}\left(z_{n+1}-z_{n}\right)+\eta_{2}\left(u_{n+1}-u_{n}\right)+\eta_{3}(1+c)\left(p_{n+1}-p_{n}\right) .
\end{aligned}
$$

Using Lemma 3.1 in [22], we get

$$
\lim _{p \rightarrow 0^{+}} \frac{f\left(s^{0}, p\right)}{f\left(s_{n+1}, p\right)}\left(s_{n+1}-s_{n}\right) \leqslant \int_{s_{n}}^{s_{n+1}} \lim _{p \rightarrow 0^{+}} \frac{f\left(s^{0}, p\right)}{f(\tau, p)} d \tau \leqslant \lim _{p \rightarrow 0^{+}} \frac{f\left(s^{0}, p\right)}{f\left(s_{n}, p\right)}\left(s_{n+1}-s_{n}\right) .
$$

Hence

$$
\Delta L_{n} \leqslant\left(1-\lim _{p \rightarrow 0^{+}} \frac{f\left(s^{0}, p\right)}{f\left(s_{n+1}, p\right)}\right)\left(s_{n+1}-s_{n}\right)+\eta_{1}\left(z_{n+1}-z_{n}\right)+\eta_{2}\left(u_{n+1}-u_{n}\right)+\eta_{3}(1+c)\left(p_{n+1}-p_{n}\right) .
$$


From Eqs. (4.1)-(4.4), we have

$$
\begin{aligned}
\Delta L_{n} \leqslant & \left(1-\lim _{p \rightarrow 0} \frac{f\left(s^{0}, p\right)}{f\left(s_{n+1}, p\right)}\right)\left(\beta-\delta s_{n+1}-f\left(s_{n+1}, p_{n}\right)\right)+\eta_{1}\left((1-\alpha) f\left(s_{n+1}, p_{n}\right)-d z_{n+1}\right) \\
& +\eta_{2}\left(\alpha f\left(s_{n+1}, p_{n}\right)-a u_{n+1}\right)+\eta_{3}\left(N_{z} d z_{n+1}+N_{u} a u_{n+1}-c p_{n+1}\right)+\eta_{3} c\left(p_{n+1}-p_{n}\right) .
\end{aligned}
$$

Collecting terms of Eq (4.15) and using $s^{0}=\frac{\beta}{\delta}$, we obtain

$$
\begin{aligned}
\Delta \mathrm{L}_{n} & \leqslant \delta s^{0}\left(1-\frac{s_{n+1}}{s^{0}}\right)\left(1-\frac{\partial f\left(s^{0}, 0\right) / \partial p}{\partial f\left(s_{n+1}, 0\right) / \partial p}\right)+\frac{\partial f\left(s^{0}, 0\right) / \partial p}{\partial f\left(s_{n+1}, 0\right) / \partial p} f\left(s_{n+1}, p_{n}\right)-\eta_{3} c p_{n} \\
& =\delta s^{0}\left(1-\frac{s_{n+1}}{s^{0}}\right)\left(1-\frac{\partial f\left(s^{0}, 0\right) / \partial p}{\partial f\left(s_{n+1}, 0\right) / \partial p}\right)+\left(\frac{\partial f\left(s^{0}, 0\right) / \partial p}{\partial f\left(s_{n+1}, 0\right) / \partial p} \frac{f\left(s_{n+1}, p_{n}\right)}{p_{n}}-\eta_{3} c\right) p_{n} .
\end{aligned}
$$

From Assumption (A4) we have

$$
\begin{aligned}
\frac{f\left(s_{n+1}, p_{n}\right)}{p_{n}} & \leqslant \lim _{p \rightarrow 0^{+}} \frac{f\left(s_{n+1}, p\right)}{p} \\
& =\frac{\partial f\left(s_{n+1}, 0\right)}{\partial p}
\end{aligned}
$$

then, we get

$$
\begin{aligned}
& =\delta s^{0}\left(1-\frac{s_{n+1}}{s^{0}}\right)\left(1-\frac{\partial f\left(s^{0}, 0\right) / \partial p}{\partial f\left(s_{n+1}, 0\right) / \partial p}\right)+\left(\frac{\partial f\left(s^{0}, 0\right)}{\partial p}-\eta_{3} c\right) p_{n} \\
& =\delta s^{0}\left(1-\frac{s_{n+1}}{s^{0}}\right)\left(1-\frac{\partial f\left(s^{0}, 0\right) / \partial p}{\partial f\left(s_{n+1}, 0\right) / \partial p}\right)+\eta_{3} c\left(\frac{\gamma}{c} \frac{\partial f\left(s^{0}, 0\right)}{\partial p}-1\right) p_{n} \\
& =\delta s^{0}\left(1-\frac{s_{n+1}}{s^{0}}\right)\left(1-\frac{\partial f\left(s^{0}, 0\right) / \partial p}{\partial f\left(s_{n+1}, 0\right) / \partial p}\right)+\eta_{3} c\left(\mathcal{R}_{0}-1\right) p_{n} .
\end{aligned}
$$

From Assumption (A3) we have

$$
\left(1-\frac{s_{n+1}}{s^{0}}\right)\left(1-\frac{\partial f\left(s^{0}, 0\right) / \partial p}{f\left(s_{n+1}, 0\right) / \partial p}\right) \leqslant 0
$$

Hence, if $\mathcal{R}_{0} \leqslant 1$, we have $\Delta \mathrm{L}_{\mathrm{n}} \leqslant 0$ for all $\mathrm{n} \geqslant 0$. Obviously, $\Delta \mathrm{L}_{\mathrm{n}}=0$ if and only if $s_{\mathrm{n}}=s^{0}$ and $\left(\mathcal{R}_{0}-1\right) p_{\mathrm{n}}=0$. We discuss two cases:

- if $\mathcal{R}_{0}<1$, then $\lim _{n \rightarrow \infty} p_{n}=0$. then we get $\lim _{n \rightarrow \infty} z_{n}=0$ and $\lim _{n \rightarrow \infty} u_{n}=0$;

- if $\mathcal{R}_{0}=1$, then by using $\lim _{n \rightarrow \infty} s_{n}=s^{0}$ and from Eq. (4.1), we obtain $f\left(s^{0}, p_{n}\right)=0$. Because $s^{0}>0$, we have $f\left(s^{0}, p_{n}\right)>f\left(0, p_{n}\right)=0$ (use Assumptions (A1) and (A2)). Thus, $\lim _{n \rightarrow \infty} p_{n}=0$.

By the aforementioned discussion, we deduce that the largest compact invariant set in $\left\{\left(s_{n}, z_{n}, u_{n}, p_{n}\right) \mid\right.$ $\left.\left(\Delta \mathrm{L}_{n}\right)=0\right\}$ is the just the singleton $\mathrm{Q}^{0}$. Therefore, $\mathrm{Q}^{0}$ is globally asymptotically stable by the LaSalle's invariance principle $[15,25]$.

Remark 4.4. Assumptions (A2) and (A4) imply that

$$
\left(\frac{f(s, p)}{p}-\frac{f\left(s, p^{*}\right)}{p^{*}}\right)\left(f(s, p)-f\left(s, p^{*}\right)\right) \leqslant 0,
$$

which yields

$$
\left(\frac{f(s, p)}{f\left(s, p^{*}\right)}-\frac{p}{p^{*}}\right)\left(1-\frac{f\left(s, p^{*}\right)}{f(s, p)}\right) \leqslant 0
$$


Theorem 4.5. Suppose that $\mathcal{R}_{0}>1$, then $\mathrm{Q}^{*}$ of system (4.1)-(4.4) is globally asymptotically stable.

Proof. Consider

$$
U_{n}\left(s_{n}, z_{n}, u_{n}, p_{n}\right)=s_{n}-s^{*}-\int_{s^{*}}^{s_{n}} \frac{f\left(s^{*}, p^{*}\right)}{f\left(\tau, p^{*}\right)} d \tau+\eta_{1} z^{*} G\left(\frac{z_{n}}{z^{*}}\right)+\eta_{2} u^{*} G\left(\frac{u_{n}}{u^{*}}\right)+\eta_{3}(1+c) p^{*} G\left(\frac{p_{n}}{p^{*}}\right) .
$$

Clearly, $u_{n}\left(s_{n}, z_{n}, u_{n}, p_{n}\right)>0$ for all $s_{n}, z_{n}, u_{n}, p_{n}>0$ and $u_{n}\left(s^{*}, z^{*}, u^{*}, p^{*}\right)=0$. Computing $\Delta u_{n}=$ $\mathrm{U}_{\mathrm{n}+1}-\mathrm{U}_{\mathrm{n}}$ as:

$$
\begin{aligned}
\Delta u_{n}= & s_{n+1}-s^{*}-\int_{s^{*}}^{s_{n+1}} \frac{f\left(s^{*}, p^{*}\right)}{f\left(\tau, p^{*}\right)} d \tau+\eta_{1} z^{*} G\left(\frac{z_{n+1}}{z^{*}}\right)+\eta_{2} u^{*} G\left(\frac{u_{n+1}}{u^{*}}\right)+\eta_{3}(1+c) p^{*} G\left(\frac{p_{n+1}}{p^{*}}\right) \\
& -\left[s_{n}-s^{*}-\int_{s_{n}^{*}}^{s_{n}} \frac{f\left(s^{*}, p^{*}\right)}{f\left(\tau, p^{*}\right)} d \tau+\eta_{1} z^{*} G\left(\frac{z_{n}}{z^{*}}\right)+\eta_{2} u^{*} G\left(\frac{u_{n}}{u^{*}}\right)+\eta_{3}(1+c) p^{*} G\left(\frac{p_{n}}{p^{*}}\right)\right] \\
= & s_{n+1}-s_{n}-\int_{s_{n}}^{s_{n+1}} \frac{f\left(s^{*}, p^{*}\right)}{f\left(\tau, p^{*}\right)} d \tau+\eta_{1} z^{*}\left[G\left(\frac{z_{n+1}}{z^{*}}\right)-G\left(\frac{z_{n}}{z^{*}}\right)\right]+\eta_{2} u^{*}\left[G\left(\frac{u_{n+1}}{u^{*}}\right)-G\left(\frac{u_{n}}{u^{*}}\right)\right] \\
& +\eta_{3}(1+c) p^{*}\left[G\left(\frac{p_{n+1}}{p^{*}}\right)-G\left(\frac{p_{n}}{p^{*}}\right)\right] .
\end{aligned}
$$

From Lemma 3.1 in [22], we have

$$
\left(1-\frac{f\left(s^{*}, p^{*}\right)}{f\left(s_{n}, p^{*}\right)}\right)\left(s_{n+1}-s_{n}\right) \leqslant s_{n+1}-s_{n}-\int_{s_{n}}^{s_{n+1}} \frac{f\left(s^{*}, p^{*}\right)}{f\left(\tau, p^{*}\right)} d \tau \leqslant\left(1-\frac{f\left(s^{*}, p^{*}\right)}{f\left(s_{n+1}, p^{*}\right)}\right)\left(s_{n+1}-s_{n}\right) .
$$

Then

$$
\begin{aligned}
\Delta u_{n} \leqslant & \left(1-\frac{f\left(s^{*}, p^{*}\right)}{f\left(s_{n+1}, p^{*}\right)}\right)\left(s_{n+1}-s_{n}\right)+\eta_{1} z^{*}\left(\frac{z_{n+1}}{z^{*}}-\frac{z_{n}}{z^{*}}+\ln \frac{z_{n}}{z_{n+1}}\right)+\eta_{2} u^{*}\left(\frac{u_{n+1}}{u^{*}}-\frac{u_{n}}{u^{*}}+\ln \frac{u_{n}}{u_{n+1}}\right) \\
& +\eta_{3} p^{*}\left(\frac{p_{n+1}}{p^{*}}-\frac{p_{n}}{p^{*}}+\ln \frac{p_{n}}{p_{n+1}}\right)+\eta_{3} c p^{*}\left[G\left(\frac{p_{n+1}}{p^{*}}\right)-G\left(\frac{p_{n}}{p^{*}}\right)\right] .
\end{aligned}
$$

Using inequality (2.7), we get

$$
\begin{aligned}
\Delta u_{n} \leqslant & \left(1-\frac{f\left(s^{*}, p^{*}\right)}{f\left(s_{n+1}, p^{*}\right)}\right)\left(s_{n+1}-s_{n}\right)+\eta_{1}\left(z_{n+1}-z_{n}+z^{*}\left(\frac{z_{n}}{z_{n+1}}-1\right)\right) \\
& +\eta_{2}\left(u_{n+1}-u_{n}+u^{*}\left(\frac{u_{n}}{u_{n+1}}-1\right)\right) \\
& +\eta_{3}\left(p_{n+1}-p_{n}+p^{*}\left(\frac{p_{n}}{p_{n+1}}-1\right)\right)+\eta_{3} c p^{*}\left(G\left(\frac{p_{n+1}}{p^{*}}\right)-G\left(\frac{p_{n}}{p^{*}}\right)\right) \\
= & \left(1-\frac{f\left(s^{*}, p^{*}\right)}{f\left(s_{n+1}, p^{*}\right)}\right)\left(s_{n+1}-s_{n}\right)+\eta_{1}\left(1-\frac{z^{*}}{z_{n+1}}\right)\left(z_{n+1}-z_{n}\right)+\eta_{2}\left(1-\frac{u^{*}}{u_{n+1}}\right)\left(u_{n+1}-u_{n}\right) \\
& +\eta_{3}\left(1-\frac{p^{*}}{p_{n+1}}\right)\left(p_{n+1}-p_{n}\right)+\eta_{3} c p^{*}\left(G\left(\frac{p_{n+1}}{p^{*}}\right)-G\left(\frac{p_{n}}{p^{*}}\right)\right) .
\end{aligned}
$$

From Eqs. (4.1)-(4.4), we have

$$
\begin{aligned}
\Delta u_{n} \leqslant & \left(1-\frac{f\left(s^{*}, p^{*}\right)}{f\left(s_{n+1}, p^{*}\right)}\right)\left(\beta-\delta s_{n+1}-f\left(s_{n+1}, p_{n}\right)\right)+\eta_{1}\left(1-\frac{z^{*}}{z_{n+1}}\right)\left((1-\alpha) f\left(s_{n+1}, p_{n}\right)-d z_{n+1}\right) \\
& +\eta_{2}\left(1-\frac{u^{*}}{u_{n+1}}\right)\left(\alpha f\left(s_{n+1}, p_{n}\right)-a u_{n+1}\right)+\eta_{3}\left(1-\frac{p^{*}}{p_{n+1}}\right)\left(N_{z} d z_{n+1}+N_{u} a u_{n+1}-c p_{n+1}\right) \\
& +\eta_{3} c\left(p_{n+1}-p_{n}\right)+\eta_{3} c p^{*} \ln \frac{p_{n}}{p_{n+1}}
\end{aligned}
$$




$$
\begin{aligned}
= & \left(1-\frac{f\left(s^{*}, p^{*}\right)}{f\left(s_{n+1}, p^{*}\right)}\right)\left(\beta-\delta s_{n+1}\right)+\frac{f\left(s^{*}, p^{*}\right)}{f\left(s_{n+1}, p^{*}\right)} f\left(s_{n+1}, p_{n}\right)-\eta_{1} \frac{z^{*}}{z_{n+1}}(1-\alpha) f\left(s_{n+1}, p_{n}\right)+\eta_{1} d z^{*} \\
& -\eta_{2} \frac{u^{*}}{u_{n+1}} \alpha f\left(s_{n+1}, p_{n}\right)+\eta_{2} a u^{*}-\eta_{3} \frac{p^{*}}{p_{n+1}}\left(N_{z} d z_{n+1}+N_{u} a u_{n+1}\right)+\eta_{3} c p^{*}-\eta_{3} c p_{n}+\eta_{3} c p^{*} \ln \frac{p_{n}}{p_{n+1}} .
\end{aligned}
$$

Using the conditions of $\mathrm{Q}^{*}$,

$$
\beta=\delta s^{*}+f\left(s^{*}, p^{*}\right), \quad(1-\alpha) f\left(s^{*}, p^{*}\right)=d z^{*}, \quad \alpha f\left(s^{*}, p^{*}\right)=a u^{*}, \quad N_{z} d z^{*}+N_{u} a u^{*}=c p^{*},
$$

we get

$$
f\left(s^{*}, p^{*}\right)=\eta_{1} d z^{*}+\eta_{2} a u^{*}=\eta_{3} c p^{*}
$$

and

$$
\begin{aligned}
& \Delta u_{n} \leqslant\left(1-\frac{f\left(s^{*}, p^{*}\right)}{f\left(s_{n+1}, p^{*}\right)}\right)\left(\delta s^{*}+f\left(s^{*}, p^{*}\right)-\delta s_{n+1}\right)+\frac{f\left(s^{*}, p^{*}\right)}{f\left(s_{n+1}, p^{*}\right)} f\left(s_{n+1}, p_{n}\right) \\
& -\eta_{1}(1-\alpha) f\left(s^{*}, p^{*}\right) \frac{f\left(s_{n+1}, p_{n}\right)}{f\left(s^{*}, p^{*}\right)} \frac{z^{*}}{z_{n+1}}+\eta_{1} d z^{*}-\eta_{2} \alpha f\left(s^{*}, p^{*}\right) \frac{f\left(s_{n+1}, p_{n}\right)}{f\left(s^{*}, p^{*}\right)} \frac{u^{*}}{u_{n+1}}+\eta_{2} a u^{*} \\
& -\eta_{1} d z^{*} \frac{z_{n+1} p^{*}}{z^{*} p_{n+1}}-\eta_{2} a u^{*} \frac{u_{n+1} p^{*}}{u^{*} p_{n+1}}+\eta_{1} d z^{*}+\eta_{2} a u^{*}-f\left(s^{*}, p^{*}\right) \frac{p_{n}}{p_{*}}+\eta_{3} c p^{*} \ln \frac{p_{n}}{p_{n+1}} \\
& =\delta s^{*}\left(1-\frac{s_{n+1}}{s^{*}}\right)\left(1-\frac{f\left(s^{*}, p^{*}\right)}{f\left(s_{n+1}, p^{*}\right)}\right)+\left(\eta_{1} d z^{*}+\eta_{2} a u^{*}\right)\left(1-\frac{f\left(s^{*}, p^{*}\right)}{f\left(s_{n+1}, p^{*}\right)}\right)+f\left(s^{*}, p^{*}\right) \frac{f\left(s_{n+1}, p_{n}\right)}{f\left(s_{n+1}, p^{*}\right)} \\
& -\eta_{1} d z^{*} \frac{f\left(s_{n+1}, p_{n}\right)}{f\left(s^{*}, p^{*}\right)} \frac{z^{*}}{z_{n+1}}+\eta_{1} d z^{*}-\eta_{2} a u^{*} \frac{f\left(s_{n+1}, p_{n}\right)}{f\left(s^{*}, p^{*}\right)} \frac{u^{*}}{u_{n+1}}+\eta_{2} a u^{*} \\
& -\eta_{1} d z^{*} \frac{z_{n+1} p^{*}}{z^{*} p_{n+1}}-\eta_{2} a u^{*} \frac{u_{n+1} p^{*}}{u^{*} p_{n+1}}+\eta_{1} d z^{*}+\eta_{2} a u^{*}-f\left(s^{*}, p^{*}\right) \frac{p_{n}}{p_{*}}+\eta_{3} c p^{*} \ln \frac{p_{n}}{p_{n+1}}, \\
& \Delta u_{n} \leqslant \delta s^{*}\left(1-\frac{f\left(s^{*}, p^{*}\right)}{f\left(s_{n+1}, p^{*}\right)}\right)\left(1-\frac{s_{n+1}}{s^{*}}\right)+f\left(s^{*}, p^{*}\right)\left[-1+\frac{p_{n} f\left(s_{n+1}, p^{*}\right)}{p^{*} f\left(s_{n+1}, p_{n}\right)}+\frac{f\left(s_{n+1}, p_{n}\right)}{f\left(s_{n+1}, p^{*}\right)}-\frac{p_{n}}{p^{*}}\right] \\
& +\eta_{1} d z^{*}\left[4-\frac{f\left(s^{*}, p^{*}\right)}{f\left(s_{n+1}, p^{*}\right)}-\frac{f\left(s_{n+1}, p_{n}\right)}{f\left(s^{*}, p^{*}\right)} \frac{z^{*}}{z_{n+1}}-\frac{z_{n+1} p^{*}}{z^{*} p_{n+1}}-\frac{p_{n} f\left(s_{n+1}, p^{*}\right)}{p^{*} f\left(s_{n+1}, p_{n}\right)}+\ln \frac{p_{n}}{p_{n+1}}\right] \\
& +\eta_{2} a u^{*}\left[4-\frac{f\left(s^{*}, p^{*}\right)}{f\left(s_{n+1}, p^{*}\right)}-\frac{f\left(s_{n+1}, p_{n}\right)}{f\left(s^{*}, p^{*}\right)} \frac{u^{*}}{u_{n+1}}-\frac{u_{n+1} p^{*}}{u^{*} p_{n+1}}-\frac{p_{n} f\left(s_{n+1}, p^{*}\right)}{p^{*} f\left(s_{n+1}, p_{n}\right)}+\ln \frac{p_{n}}{p_{n+1}}\right] \\
& =\delta s^{*}\left(1-\frac{f\left(s^{*}, p^{*}\right)}{f\left(s_{n+1}, p^{*}\right)}\right)\left(1-\frac{s_{n+1}}{s^{*}}\right)+f\left(s^{*}, p^{*}\right)\left[-1+\frac{p_{n} f\left(s_{n+1}, p^{*}\right)}{p^{*} f\left(s_{n+1}, p_{n}\right)}+\frac{f\left(s_{n+1}, p_{n}\right)}{f\left(s_{n+1}, p^{*}\right)}-\frac{p_{n}}{p^{*}}\right] \\
& -\eta_{1} d z^{*}\left[G\left(\frac{f\left(s^{*}, p^{*}\right)}{f\left(s_{n+1}, p^{*}\right)}\right)+G\left(\frac{f\left(s_{n+1}, p_{n}\right)}{f\left(s^{*}, p^{*}\right)} \frac{z^{*}}{z_{n+1}}\right)+G\left(\frac{z_{n+1} p^{*}}{z^{*} p_{n+1}}\right)+G\left(\frac{p_{n} f\left(s_{n+1}, p^{*}\right)}{p^{*} f\left(s_{n+1}, p_{n}\right)}\right)\right] \\
& -\eta_{2} a u^{*}\left[G\left(\frac{f\left(s^{*}, p^{*}\right)}{f\left(s_{n+1}, p^{*}\right)}\right)+G\left(\frac{f\left(s_{n+1}, p_{n}\right)}{f\left(s^{*}, p^{*}\right)} \frac{u^{*}}{u_{n+1}}\right)+G\left(\frac{u_{n+1} p^{*}}{u^{*} p_{n+1}}\right)+G\left(\frac{p_{n} f\left(s_{n+1}, p^{*}\right)}{p^{*} f\left(s_{n+1}, p_{n}\right)}\right)\right] \text {. }
\end{aligned}
$$

Because $f(s, p)$ is strictly increasing with respect to $s$, we obtain that

$$
\left(1-\frac{f\left(s^{*}, p^{*}\right)}{f\left(s_{n+1}, p^{*}\right)}\right)\left(1-\frac{s_{n+1}}{s^{*}}\right) \leqslant 0 .
$$

Based on Remark 4.4, we have

$$
-1-\frac{p_{n}}{p^{*}}+\frac{p_{n} f\left(s_{n+1}, p^{*}\right)}{p^{*} f\left(s_{n+1}, p_{n}\right)}+\frac{f\left(s_{n+1}, p_{n}\right)}{f\left(s_{n+1}, p^{*}\right)}=\left(1-\frac{f\left(s_{n+1}, p^{*}\right)}{f\left(s_{n+1}, p_{n}\right)}\right)\left(\frac{f\left(s_{n+1}, p_{n}\right)}{f\left(s_{n+1}, p^{*}\right)}-\frac{p_{n}}{p^{*}}\right) \leqslant 0 .
$$

Thus, $U_{n}$ is monotone decreasing sequence. Because $u_{n} \geqslant 0$, there is a limit $\lim _{n \rightarrow \infty} u_{n} \geqslant 0$. Therefore, $\lim _{n \rightarrow \infty} \Delta U_{n}=0$, which implies that $\lim _{n \rightarrow \infty} s_{n}=s^{*}, \lim _{n \rightarrow \infty} z_{n}=z^{*}, \lim _{n \rightarrow \infty} u_{n}=u^{*}$ and $\lim _{n \rightarrow \infty} p_{n}=p^{*}$. 


\section{Numerical simulations}

We perform our simulation by choosing Crowly Martin incidence rate

$$
f(s, p)=\frac{k s p}{(1+\lambda s)(1+\theta p)},
$$

where $\lambda>0$ and $\theta>0$. Therefore, system (4.1)-(4.4) becomes

$$
\begin{aligned}
s_{n+1}-s_{n} & =\beta-\delta s_{n+1}-\frac{(1-\epsilon) \bar{k} s_{n+1} p_{n}}{\left(1+\lambda s_{n+1}\right)\left(1+\theta p_{n}\right)}, \\
z_{n+1}-z_{n} & =\frac{(1-\alpha)(1-\epsilon) \bar{k} s_{n+1} p_{n}}{\left(1+\lambda s_{n+1}\right)\left(1+\theta p_{n}\right)}-d z_{n+1}, \\
u_{n+1}-u_{n} & =\frac{\alpha \bar{k} s_{n+1} p_{n}}{\left(1+\lambda s_{n+1}\right)\left(1+\theta p_{n}\right)}-a u_{n+1}, \\
p_{n+1}-p_{n} & =N_{z} d z_{n+1}+N_{u} a u_{n+1}-c p_{n+1} .
\end{aligned}
$$

For this system, the basic reproduction number is given by

$$
\mathcal{R}_{0}=\frac{\gamma(1-\epsilon) \bar{k} s^{0}}{c\left(1+\lambda s^{0}\right)} .
$$

We verify the assumptions (A1)-(A4) as:

$$
\begin{aligned}
f(s, p) & =\frac{(1-\epsilon) \bar{k} s p}{(1+\lambda s)(1+\theta p)}>0, \text { and } f(0, p)=f(s, 0)=0 \text { for all } s>0, p>0, \\
\frac{\partial f(s, p)}{\partial s} & =\frac{(1-\epsilon) \bar{k} p}{(1+\theta p)(1+\lambda s)^{2}}>0 \text { for all } s>0, \text { and } p>0, \\
\frac{\partial f(s, p)}{\partial p} & =\frac{(1-\epsilon) \bar{k} s}{(1+\lambda s)(1+\theta p)^{2}} \text { for all } s>0, \text { and } p>0, \\
\frac{\partial f(s, 0)}{\partial p} & =\frac{(1-\epsilon) \bar{k} s}{1+\lambda s}>0 \text { for all } s>0, \\
\frac{d}{d s}\left(\frac{\partial f(s, 0)}{\partial p}\right) & =\frac{(1-\epsilon) \bar{k}}{(1+\lambda s)^{2}}>0 \text { for all } s>0, \\
\frac{d}{d p}\left(\frac{f(s, p)}{p}\right) & =\frac{-\theta(1-\epsilon) \bar{k} s}{(1+\lambda s)(1+\theta p)^{2}}<0 \text { for all } s>0, \text { and } p>0 .
\end{aligned}
$$

Then, function $f(s, p)$ satisfies Assumptions (A1)-(A4) and hence Theorems 4.3 and 4.5 are applicable for such function.

Remark 5.1. We note that Assumptions (A1)-(A4) can also be satisfied for other types of the incidence rate function such as: Beddington-DeAngelis incidence $f(s, p)=\frac{k s p}{1+\lambda s+\theta p}$ and Hill-type incidence $f(s, p)=$ $\frac{k s^{m} p}{\lambda^{m}+s^{m}}, m>0$.

The numerical simulations for system (5.1)-(5.4) will be conducted using the following data: $\beta=10$, $\delta=0.01, \alpha=0.5, \mathrm{~d}=0.3, \mathrm{a}=0.25, \mathrm{c}=2, \mathrm{~N}_{z}=10, \mathrm{~N}_{\mathfrak{u}}=5, \lambda=0.0001$ and $\theta=0.0001$. The other parameters will be chosen as bellow.

Let us consider the initial values

IV1: $s(0)=800, w(0)=10, u(0)=20, p(0)=20$,

IV2: $s(0)=600, w(0)=2, u(0)=15, p(0)=15$,

IV3: $s(0)=400, w(0)=5, u(0)=10, p(0)=10$.

\section{Case (I) Effect of $\bar{k}$ of stability of equilibria:}

We choose $\epsilon=0.5$ and $\bar{k}$ is varied as: 
(i) $\bar{k}=0.0001$. This yields $\mathcal{R}_{0}=0.1705<1$. Figures $1-4$ show that, the concentration of susceptible cells increases and tends to the value $s^{0}=1000$. In addition, the concentrations of long-lived infected cells, short-lived infected cells and free HIV decrease and tend to zero for the initial values IV1-IV3. This shows that $\mathrm{Q}^{0}$ is globally asymptotically stable and Theorem 4.3 is valid.

(ii) $\bar{k}=0.001$. With this value we obtain $\mathcal{R}_{0}=1.7045>1$. Figures $1-4$ show that for the initial values IV1-IV3, the solutions of the system tend to the equilibrium $\mathrm{Q}^{*}=(564.4143,7.2953,8.7541,16.4141)$. Therefore, $\mathrm{Q}^{*}$ exists and it is globally asymptotically stable. This validate the result of Theorem 4.5.

\section{Case (II) Effect of the drug efficacy $\epsilon$ on the HIV dynamics:}

For this case, we take IV2 and choose the value $\bar{k}=0.001$ and $\epsilon$ is varied. Figures 5-8 and Table 1 show the effect of drug efficacy $\epsilon$ on the stability of the system. We observe that, as $\epsilon$ is increased, the infection rate is decreased, then, the concentration of the susceptible cells are increased, while the concentrations of the long-lived infected cells, short-lived infected cells and free HIV particles are decreased. In addition

(i) if $\epsilon<0.70666$, then $\mathrm{Q}^{*}$ is globally asymptotically stable;

(ii) if $\epsilon \geqslant 0.70666$, then $\mathrm{Q}^{0}$ is globally asymptotically stable.

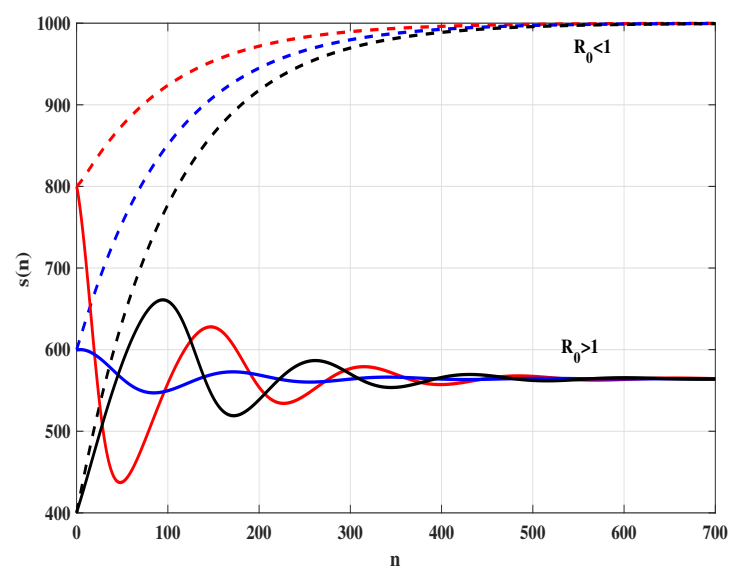

Figure 1: The simulation of susceptible cells of system (5.1)-(5.4) for Case (I).

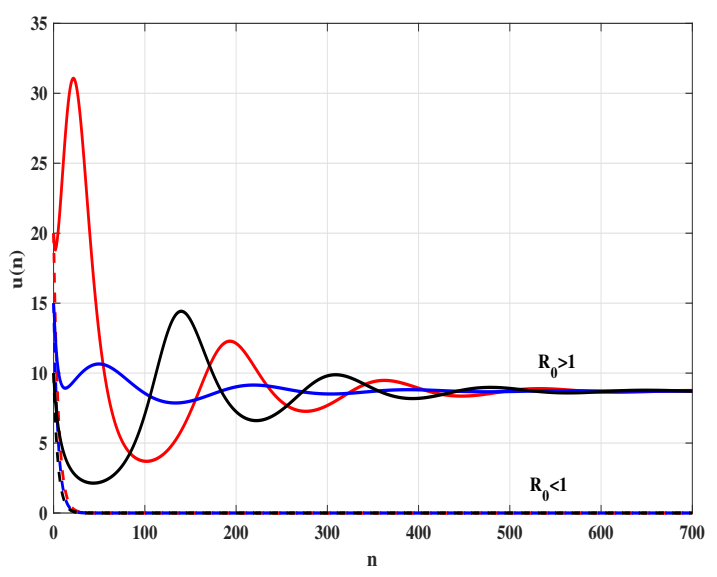

Figure 3: The simulation of long-lived infected cells of system (5.1)-(5.4) for Case (I).

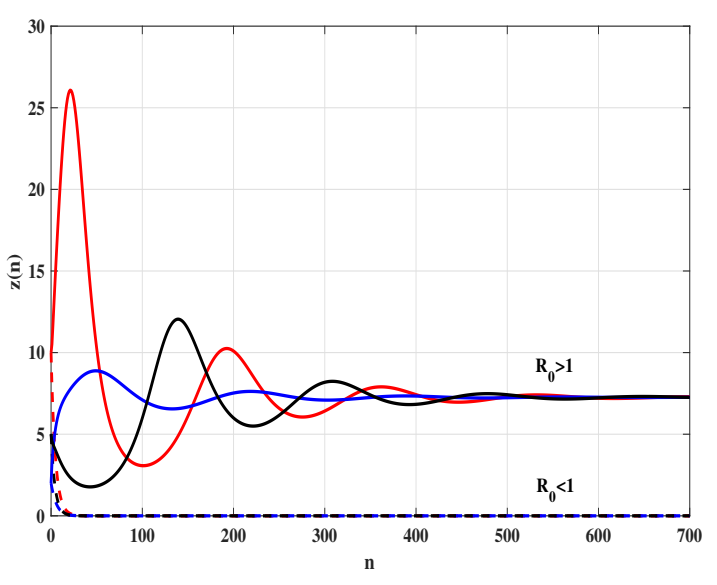

Figure 2: The simulation of short-lived infected cells of system (5.1)-(5.4) for Case (I).

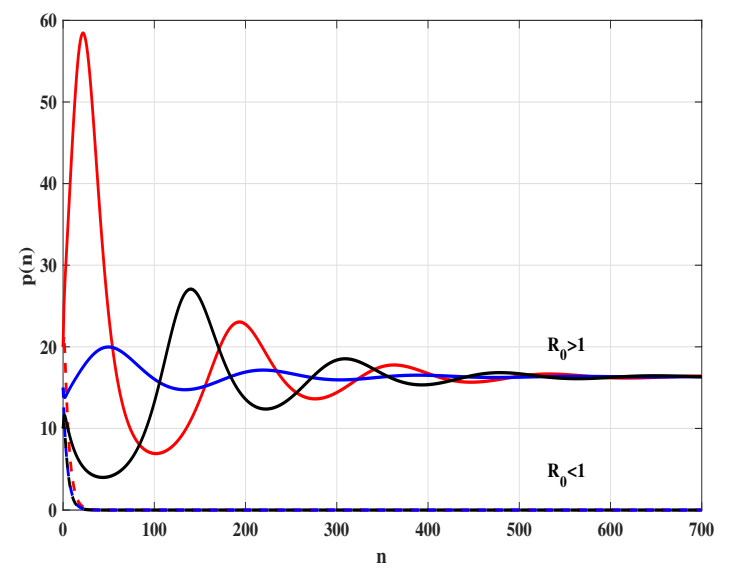

Figure 4: The simulation of pathogens of system (5.1)-(5.4) for Case (I). 


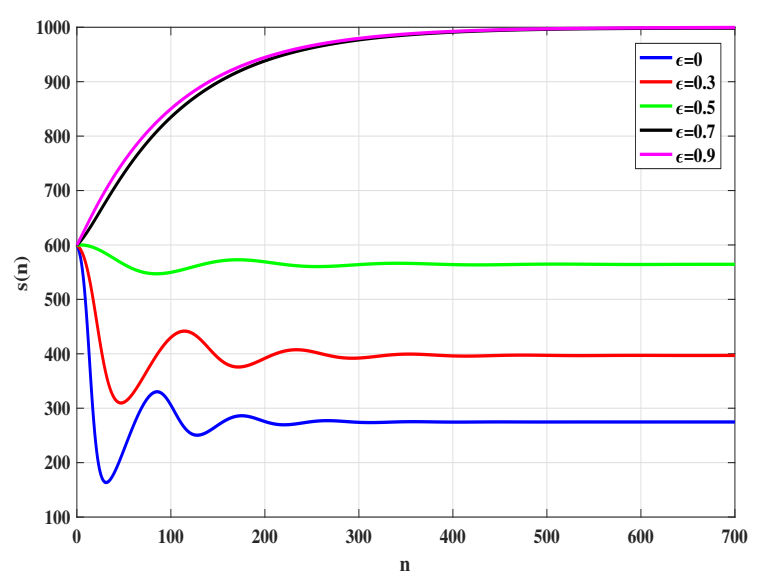

Figure 5: The simulation of susceptible cells of system (5.1)-(5.4) for Case (II).

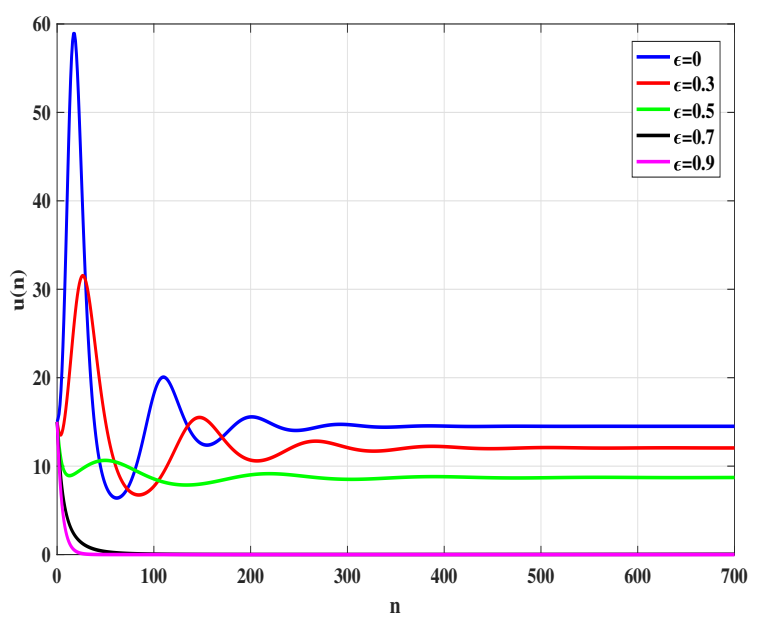

Figure 7: The simulation of long-lived infected cells of system (5.1)-(5.4) for Case (II).

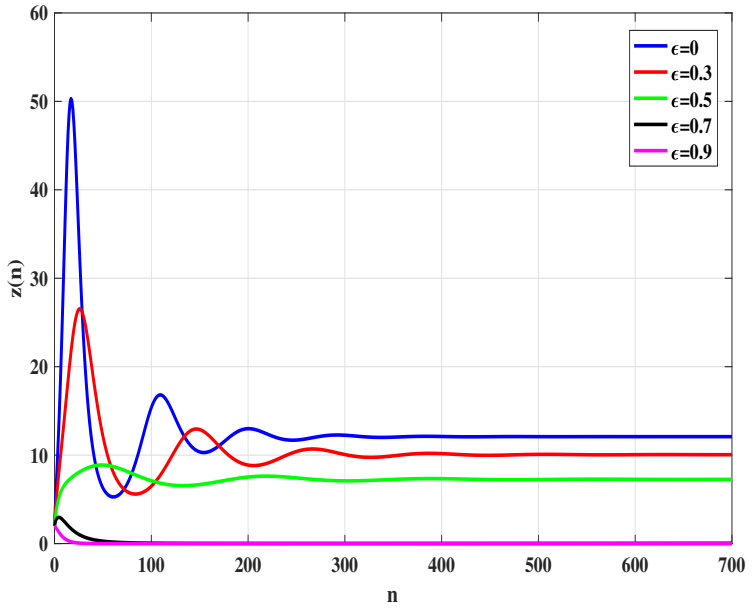

Figure 6: The simulation of short-lived infected cells of system (5.1)-(5.4) for Case (II).

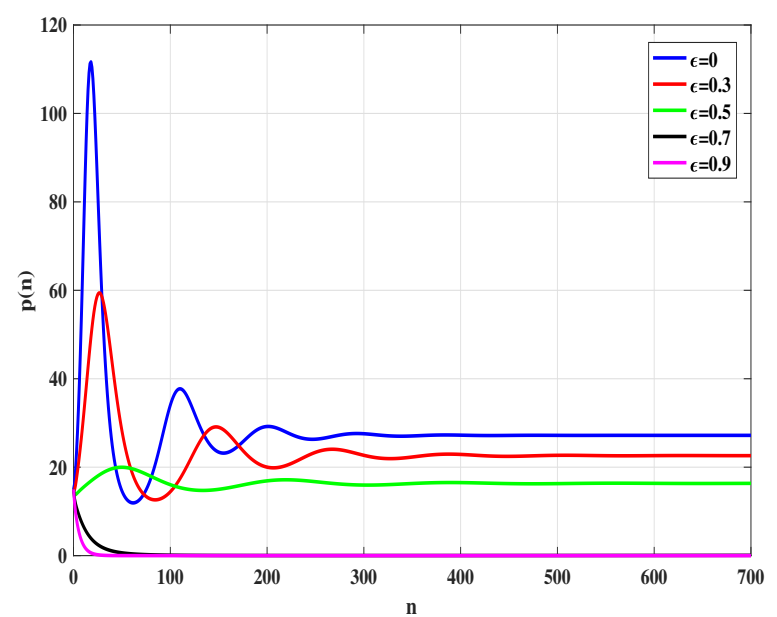

Figure 8: The simulation of pathogens of system (5.1)-(5.4) for Case (II).

Table 1: The values of $\mathcal{R}_{0}$ for system (5.1)-(5.4) with different values of $\epsilon$.

\begin{tabular}{ccc}
\hline$\epsilon$ & Equilibria & $\mathcal{R}_{0}$ \\
\hline 0 & $\mathrm{Q}^{*}$ & 3.4091 \\
0.3 & $\mathrm{Q}^{*}$ & 2.3864 \\
0.5 & $\mathrm{Q}^{*}$ & 1.7045 \\
0.7 & $\mathrm{Q}^{*}$ & 1.0227 \\
0.70666 & $\mathrm{Q}^{0}$ & 1 \\
0.8 & $\mathrm{Q}^{0}$ & 0.6818 \\
0.9 & $\mathrm{Q}^{0}$ & 0.3409 \\
\hline
\end{tabular}

\section{References}

[1] D. S. Callaway, A. S. Perelson, HIV-1 infection and low steady state viral loads, Bull. Math. Biol., 64 (2002), 29-64. 1, 1

[2] R. V. Culshaw, S. G. Ruan, A delay-differential equation model of HIV infection of CD4 ${ }^{+}$T-cells, Math. Biosci., 165 (2000), 27-39. 
[3] R. V. Culshaw, S. G. Ruan, G. Webb, A mathematical model of cell-to-cell spread of HIV-1 that includes a time delay, J. Math. Biol., 46 (2003), 425-444. 1

[4] D. Q. Ding, X. H. Ding, Dynamic consistent non-standard numerical scheme for a dengue disease transmission model, J. Difference Equ. Appl., 20 (2014), 492-505. 1

[5] D. Ding, W. Qin, X. Ding, Lyapunov functions and global stability for a discretized multigroup SIR epidemic model, Discrete Contin. Dyn. Syst. Ser. B, 20 (2015), 1971-1981. 1

[6] A. Dutta , P. K. Gupta, A mathematical model for transmission dynamics of HIV/AIDS with effect of weak CD4 ${ }^{+} \mathrm{T}$ cells, Chinese J. Phys., 56 (2018), 1045-1056. 1

[7] A. M. Elaiw, Global properties of a class of HIV models, Nonlinear Anal. Real World Appl., 11 (2010), $2253-2263$.

[8] A. M. Elaiw, Global properties of a class of virus infection models with multitarget cells, Nonlinear Dynam., 69 (2012), 423-435.

[9] A. M. Elaiw, N. A. Almuallem, Global properties of delayed-HIV dynamics models with differential drug efficacy in cocirculating target cells, Appl. Math. Comput., 265 (2015), 1067-1089.

[10] A. M. Elaiw, N. A. Almuallem, Global dynamics of delay-distributed HIV infection models with differential drug efficacy in cocirculating target cells, Math. Methods Appl. Sci., 39 (2016), 4-31.

[11] A. M. Elaiw, N. H. AlShamrani, Stability of a general delay-distributed virus dynamics model with multi-staged infected progression and immune response, Math. Methods Appl. Sci., 40 (2017), 699-719.

[12] A. M. Elaiw, I. A. Hassanien, S. A. Azoz, Global stability of HIV infection models with intracellular delays, J. Korean Math. Soc., 49 (2012), 779-794.

[13] A. M. Elaiw, A. A. Raezah, Stability of general virus dynamics models with both cellular and viral infections and delays, Math. Methods Appl. Sci., 40 (2017), 5863-5880.

[14] A. M. Elaiw, S. A. Azoz, Global properties of a class of HIV infection models with Beddington-DeAngelis functional response, Math. Methods Appl. Sci., 36 (2013), 383-394. 1

[15] S. Elaydi, An introduction to Difference Equations: Third ed., Springer, New York, (2005). 4.2

[16] Y. Enatsu, Y. Nakata, Y. Muroya, G. Izzo, A. Vecchio, Global dynamics of difference equations for SIR epidemic models with a class of nonlinear incidence rates, J. Difference Equ. Appl., 18 (2012), 1163-1181. 1

[17] Y. Geng, J. H. Xu, J. Y. Hou, Discretization and dynamic consistency of a delayed and diffusive viral infection model, Appl. Math. Comput., 316 (2018), 282-295. 1

[18] K. Hattaf, A. A. Lashari, B. El Boukari, N. Yousfi, Effect of discretization on dynamical behavior in an epidemiological model, Differ. Equ. Dyn. Syst., 23 (2015), 403-413. 1

[19] K. Hattaf, N. Yousfi, A generalized virus dynamics model with cell-to-cell transmission and cure rate, Adv. Difference Equ., 2016 (2016), 11 pages. 1

[20] K. Hattaf, N. Yousfi, A numerical method for delayed partial differential equations describing infectious diseases, Comput. Math. Appl., 72 (2016), 2741-2750. 1

[21] K. Hattaf, N. Yousfi, A numerical method for a delayed viral infection model with general incidence rate, J. King Saud Uni.-Sci., 28 (2016), 368-374.

[22] K. Hattaf, N. Yousfi, Global properties of a discrete viral infection model with general incidence rate, Math. Methods Appl. Sci., 39 (2016), 998-1004. 1, 4.2, 4.2

[23] G. Huang, Y. Takeuchi, W. Ma, Lyapunov functionals for delay differential equations model of viral infections, SIAM J. Appl. Math., 70 (2010), 2693-2708. 1

[24] A. Korpusik, A nonstandard finite difference scheme for a basic model of cellular immune response to viral infection, Commun. Nonlinear Sci. Numer. Simul., 43 (2017), 369-384. 1

[25] J. P. Lasalle, The stability and control of discrete processes, Springer-Verlag, New York, (1986). 4.2

[26] B. Li, Y. M. Chen, X. J. Lu, S. Q. Liu, A delayed HIV-1 model with virus waning term, Math. Biosci. Eng., 13 (2016), 135-157. 1

[27] M. Y. Li, L. C. Wang, Backward bifurcation in a mathematical model for HIV infection in vivo with anti-retroviral treatment, Nonlinear Anal. Real World Appl., 17 (2014), 147-160. 1

[28] J. L. Liu, B. Y. Peng, T. L. Zhang, Effect of discretization on dynamical behavior of SEIR and SIR models with nonlinear incidence, Appl. Math. Lett., 39 (2015), 60-66. 1

[29] K. Manna, S. P. Chakrabarty, Global stability and a non-standard finite difference scheme for a diffusion driven HBV model with capsids, J. Difference Equ. Appl., 21 (2015), 918-933. 1

[30] R. E. Mickens, Nonstandard Finite Difference Models of Differential equations, World Scientific Publishing Co., River Edge, (1994). 1, 2

[31] R. E. Mickens, Dynamics consistency: a fundamental principle for constructing nonstandard finite difference scheme for differential equation, J. Difference Equ. Appl., 11 (2005), 645-653. 2

[32] P. W. Nelson, J. D. Murray, A. S. Perelson, A model of HIV-1 pathogenesis that includes an intracellular delay, Math. Biosci., 163 (2000), 201-215. 1

[33] M. A. Nowak, C. R. M. Bangham, Population dynamics of immune responses to persistent viruses, Science, 272 (1996), 74-79. 1

[34] C. M. A. Pinto, A. R. M. Carvalho, A latency fractional order model for HIV dynamics, J. Comput. Appl. Math., 312 (2017), 240-256.1 
[35] W. D. Qin, L. S. Wang, X. H. Ding, A non-standard finite difference method for a hepatitis $b$ virus infection model with spatial diffusion, J. Difference Equ. Appl., 20 (2014), 1641-1651. 1

[36] P. L. Shi, L. Z. Dong, Dynamical behaviors of a discrete HIV-1 virus model with bilinear infective rate, Math. Methods Appl. Sci., 37 (2014), 2271-2280. 1, 2.1

[37] X. Y. Shi, X. Y. Zhou, X. Y. Song, Dynamical behavior of a delay virus dynamics model with CTL immune response, Nonlinear Anal. Real World Appl., 11 (2010), 1795-1809. 3

[38] X. Y. Song, A. U. Neumann, Global stability and periodic solution of the viral dynamics, J. Math. Anal. Appl., 329 (2007), 281-297. 3

[39] Z. D. Teng, L. Wang, L. F. Nie, Global attractivity for a class of delayed discrete SIRS epidemic models with general nonlinear incidence, Math. Methods Appl. Sci., 38 (2015), 4741-4759. 1

[40] L. C. Wang, M. Y. Li, Mathematical analysis of the global dynamics of a model for HIV infection of CD4 ${ }^{+} \mathrm{T}$ cells, Math. Biosci., 200 (2006), 44-57. 1

[41] J. P. Wang, Z. D. Teng, H. Miao, Global dynamics for discrete-time analog of viral infection model with nonlinear incidence and CTL immune response, Adv. Difference Equ., 2016 (2016), 19 pages. 1, 1

[42] J. Xu, Y. Geng, J. Hou, A non-standard finite difference scheme for a delayed and diffusive viral infection model with general nonlinear incidence rate, Comput. Math. Appl., 74 (2017), 1782-1798.

[43] J. H. Xu, J. Y. Hou, Y. Geng, S. X. Zhang, Dynamic consistent NSFD scheme for a viral infection model with cellular infection and general nonlinear incidence, Adv. Difference Equ., 2018 (2018), 17 pages.

[44] Y. Yang, X. S. Ma, Y. H. Li, Global stability of a discrete virus dynamics model with Holling type-II infection function, Math. Methods Appl. Sci., 39 (2016), 2078-2082.

[45] Y. Yang, J. L. Zhou, Global stability of a discrete virus dynamics model with diffusion and general infection function, Int. J. Comput. Math., 2018 (2018), 11 pages.

[46] Y. Yang, J. L. Zhou, X. S. Ma, T. H. Zhang, Nonstandard finite difference scheme for a diffusive within-host virus dynamics model both virus-to-cell and cell-to-cell transmissions, Comput. Math. Appl., 72 (2016), 1013-1020. 1

[47] Y. Zhao, D. T. Dimitrov, H. Liu, Y. Kuang, Mathematical insights in evaluating state dependent effectiveness of HIV prevention interventions, Bull. Math. Biol., 75 (2013), 649-675. 1

[48] J. L. Zhou, Y. Yang, Global dynamics of a discrete viral infection model with time delay, virus-to-cell and cell-to-cell transmissions, J. Difference Equ. Appl., 23 (2017), 1853-1868. 1 